\title{
Transcription Factor RFX3 Stabilizes Mammary Basal Cell Identity
}

2

3

10 Correspondence to:

11 lan Macara

12 Email: lan.g.macara@vanderbilt.edu

13 Tel: 615-875-5565

14

15

16

17

18

19

20

21

22

23

24

25

26

27

28

29

30

31

32 


\section{ABSTRACT}

The myoepithelial cell compartment of the murine postnatal mammary gland is generated from basal cap cells in the terminal end bud and maintained by self-renewal. Transdifferentiation to the luminal lineage does not normally occur but can be induced by DNA damage, luminal cell death or transplantation into a recipient mammary fat pad. Myoepithelial cells cultivated in vitro can also transdifferentiate towards the luminal lineage. Little is known about the molecular mechanisms and gene regulatory networks underlying this plasticity. Using a transgenic mouse (Tg11.5kb-GFP) that marks cap cells with GFP, we discovered that mature myoepithelial cells placed in culture begin to express GFP within $\sim 24$ hrs and later express the Keratin 8 (K8) luminal marker. Cell tracking showed that most K8+ cells arose from GFP+ cells, suggesting that myoepithelial cells de-differentiate towards a progenitor state before changing lineage. Differential gene expression analysis, comparing pure GFP+ cap cells with mature myoepithelial cells, identified multiple transcription factors that iRegulon predicted might regulate the myoepithelial to cap cell transition. Knockout of one of these genes, Regulatory Factor 3 (Rfx3), significantly reduced the population of GFP+ cells and increased differentiation to the K8+ luminal lineage. Rfx3 knockout also reduced mammosphere growth and mammary gland regeneration efficiency in a transplantation assay, but had no effect on proliferation in vitro. Together, these data support a key role for Rfx3 in the stabilization of the mammary basal cell lineages.

\section{INTRODUCTION}

The mammary gland undergoes dynamic changes that contribute to the development, structure and function of the organ. In utero, development is initiated by bipotent mammary stem cells that give rise to the mammary rudiment, which remains quiescent until puberty (Spike et al., 2012; Van Keymeulen et al., 2011; Wuidart et al., 2018). At the onset of puberty, the estrus cycle drives expansion of the rudiment into a branching network of ducts that fill the mammary fat pad (Silberstein, 2001). Mature ducts consist of an inner layer of polarized luminal cells (which express the keratin K8), surrounded by spindle-shaped basal, myoepithelial cells that are marked by K14 expression. Outgrowth occurs primarily from terminal end buds at the tip of each duct, filled with immature, proliferating luminal body cells enveloped by a single basal layer of cap cells, which are a metastable population of myoepithelial progenitors (Abdul-Manan et al., 1999; Humphreys et al., 1996; Sreekumar et al., 2017). These large end buds, and the cap cells, disappear when the ducts reach the edges of the fat pad (Hinck \& Silberstein, 2005). At 
self-renewal (Prater et al., 2014; Van Keymeulen et al., 2011; Wuidart et al., 2016). Each population appears to be stable; however, we discovered that DNA damage to the mammary gland triggers transdifferentiation of myoepithelial to luminal cells (Seldin and Macara 2020). Ablation of luminal cells in situ by diphtheria toxin also activates transdifferentiation, possibly through multiple signaling pathways (Centonze et al., 2020; Le Guelte, 2021). Interestingly, this same lineage switch appears to be induced by cultivating myoepithelial cells in vitro or by their transplantation into cleared mammary fat pads in a recipient mouse. However, almost nothing is known about the underlying mechanisms and gene regulatory networks that determine the stability of the lineages or their reversion to a stem-like state. To begin to untangle the processes driving myoepithelial cell transdifferentiation, we employed a transgenic mouse strain that expresses GFP from the promoter of the s-SHIP gene (11.5kb-GFP), which is expressed in embryonic and hematopoietic stem cells, and several other progenitor/stem cell lineages (Rohrschneider, Custodio, Anderson, Miller, \& Gu, 2005). It also marks cap cells but is silent in mature myoepithelial cells (Bai \& Rohrschneider, 2010). After plating purified myoepithelial cells in culture, we detected both GFP+ cells and cells expressing the luminal marker K8 arising within $24-48 \mathrm{hrs}$. Single cell RNAseq also identified a population of luminal cells after $96 \mathrm{hrs}$ culture. Cell tracking revealed that most $\mathrm{K} 8+$ cells arise from the GFP+ population, suggesting that myoepithelial cells de-differentiate before undergoing transdifferentiation towards the luminal lineage. Bulk RNAseq of purified cap cells (GFP+) and mature myoepithelial cells identified several transcription factors that were preferentially expressed in the cap cell population, which by iRegulon analysis are predicted to control multiple other genes induced in the cap cells. For one such factor, Rfx3 (regulatory factor 3 ), its ablation by CRISPR-Cas 9 editing in mature myoepithelial cells caused a significant reduction in the frequency of GFP+ cell formation, with increased formation of K8+ cells. Rfx3 knockout also reduced mammosphere formation and reduced ductal outgrowth after transplantation. Rfx3 ablation in pure cap cells also increased the formation of $\mathrm{K} 8+$ cells. We propose that $\mathrm{Rf} \times 3$ normally stabilizes basal cell identities, so that in its absence the myoepithelial and cap cell populations more rapidly convert to the luminal lineage, thereby losing the ability to regenerate mammary glands in transplantation assays. 


\section{RESULTS}

Myoepithelial cells in culture can transdifferentiate towards cap cell and luminal cell lineages

Lineage-restricted mammary myoepithelial cells in situ can transdifferentiate into luminal cells in response to DNA damage or DTA-mediated ablation of the luminal population, and are capable of regenerating entire mammary glands after transplantation into the fat pads of recipient mice (Bai \& Rohrschneider, 2010; Centonze et al., 2020; Seldin \& Macara, 2020; Stingl et al., 2006; Van Keymeulen et al., 2011). To probe mechanisms that might regulate this lineage switching, we isolated mammary glands from the $11.5 \mathrm{~kb}-$ GFP transgenic mouse, which expresses GFP specifically in cap cells of terminal end buds but not in mature myoepithelial cells (Supplementary Fig S1 A). Myoepithelial cells were then sorted by FACS (Supplementary Figure S1 B) and cultured for 96 hrs on laminin-coated cover glasses in FAD media supplemented with ROCK inhibitor (Fig $1 \mathrm{~A}$ ). Cell cultures were fixed every 24 hrs over $4 \mathrm{~d}$, stained for the luminal marker K8 and imaged to determine whether cells expressed GFP and/or K8. GFP+ cells began to appear after $24 \mathrm{hrs}$ and increased over time. K8+ cells were detectable within 48 hrs. By 96 hrs in vitro $\sim 20 \%$ of cells were GFP+, $\sim 10 \%$ of cells expressed K8 and $\sim 5 \%$ of cells were double positive (Fig $1 \mathrm{~B}, \mathrm{C}$ ). To determine if this $\mathrm{K} 8+$ population represents the true luminal lineage or is a de-differentiated cell type that happens to express this keratin but not other luminal markers, we performed single cell RNAseq (scRNAseq) (Supplementary Fig S1 C). As an initial test we purified myoepithelial, luminal and cap cells from Tg11.5kb-GFP mouse mammary glands, then pooled equal numbers of each population and processed them for inDrop scRNAseq. Uniform manifold approximation and projection (UMAP) identifies three separate clusters, as expected (Supplementary Fig S1 D). We then isolated mature myoepithelial cells and grew them in culture for $96 \mathrm{hrs}$ prior to encapsulation and scRNAseq. In this case UMAP revealed a large cluster that expresses the myoepithelial marker K14 and a separate cluster mostly positive for K8 and other luminal markers, while GFP+ cells were clustered with the K14+ population (Fig 1 D). These data suggest that the GFP+ cells do not fully de-differentiate into cap cells, but that transdifferentiation into the luminal lineage is relatively robust. We next asked if the GFP+ state is an obligatory intermediate between the myoepithelial and luminal identities, or whether different myoepithelial cells independently adopt either the luminal state or the GFP+ state. To distinguish these hypotheses, we used cell tracking. Freshly isolated myoepithelial cells from Tg11.5kb-GFP mice were plated sparsely on gridded coverslips and imaged every $12 \mathrm{hrs}$ for 96 hrs then fixed and stained for K8 (Fig 2 A,B). Each cell was then traced backwards in time to 
determine if/when cells became GFP+ and if they progressed or not to become K8+. Data were visualized as a Sankey plot (Fig $2 \mathrm{C}$ ). Of 402 cells that were analyzed, $70 \%$ began to express GFP within 72 hrs and of this population $~ 85 \%$ eventually expressed K8. In contrast, only $30 \%$ of the GFP- cells eventually expressed K8 (Fig $2 \mathrm{C}$ ). It is possible that some of these cells transiently expressed GFP and were missed during the periodic imaging. Moreover, we noted that transdifferentiation of this sparse culture of myoepithelial cells was much more efficient than occurs when the cells are plated more densely, as in Fig 1. The reason for this difference remains obscure but we can conclude, nonetheless, that the principal pathway for transdifferentiation involves an initial, partial dedifferentiation towards a progenitor, cap cell state prior to converting to the luminal lineage.

\section{RNAseq identifies multiple genes that are differentially expressed between cap cells and} mature myoepithelial cells

Myoepithelial cells and GFP+ cap cells were isolated from 5 weeks old female Tg11.5kb-GFP mice and subjected to RNAseq. Principle component analysis confirmed that like-samples had the least variance and clustered together, clearly segregating the two cell types (Supplementary Fig S2 A). We validated the RNAseq data by qRT-PCR of chosen mRNAs that were either less expressed or more highly expressed in cap cells as determined from the RNAseq (Supplementary Fig S2 B). A volcano plot identified multiple genes that were up or down regulated in the GFP+ cap cells versus myoepithelial cells (Fig 3 A). Using a p-value of $\leq 0.05$ and a fold change of $\geq 2,63$ genes were significantly upregulated in cap cells and 106 were downregulated, as compared to mature myoepithelial cells. Unsupervised hierarchical clustering also identified a cap cell gene signature corresponding to GO terms associated with organ, tissue and embryonic development, which is consistent with the status of cap cells as progenitors (Fig $3 \mathrm{~B}, \mathrm{C}$ ). TheTg11.5kb-GFP transgenic line drives GFP from an alternate promoter for the SHIP-1 (INPP5D) gene, which expresses a shorter variant of SHIP-1 called sSHIP (Rohrschneider et al., 2005). Although most of the transcribed s-SHIP sequence is identical to that of full-length SHIP-1, there is a unique 42 nt sequence in the 5' UTR that differentiates it from the canonical SHIP-1 mRNA sequence. We expected that GFP+ cells would also express this S-SHIP sequence. Indeed, analysis of the RNAseq data identified about 300 (+/- $200 \mathrm{fkpm} ; \mathrm{n=6})$ reads of the unique s-SHIP sequence in purified GFP+ cells and zero reads in the mature myoepithelial cells (Fig $3 \mathrm{D}$ ). 


\section{Transcription factors predicted to regulate the GFP+ cap cell signature}

To determine what transcription factors are regulating the expression of the genes highly expressed in GFP+ cap cells we performed Gene Regulatory Network Analysis using iRegulon, which is a computational method developed to reverse-engineer a transcriptional network underlying a co-expressed gene set using cis-regulatory sequence analysis (Janky et al., 2014). This program bases its predictions on databases of $\sim 10,000$ TF motifs and 1000 Chip-Seq data sets. The analysis identified 15 transcription factors predicted to regulate the majority of genes most highly expressed in GFP+ cap cells (Fig 4 A). We determined from the RNAseq data that of these transcription factors RFX3, MTF1, PDX1, and GATA2 are consistently upregulated in cap cells (Fig 4 B).

RFX3 is a key factor in ciliogenesis (Chen et al., 2018). Primary cilia have been reported to regulate branching morphogenesis during mammary gland development (McDermott, Liu, Tlsty, \& Pazour, 2010). It was also shown that cilia are enriched in mammospheres and cilia defects decreased the ability to form mammospheres. Loss of IFT88, a target of RFX3, reduces mammosphere growth (Mitchell \& Serra, 2014). To determine whether RFX3 is an important regulator of the conversion of myoepithelial cells into GFP+ cap cells, we used Cas9-mediated gene editing with 2 gRNAs to target and splice out the RFX3 DNA binding domain in purified mature myoepithelial cells from the Tg11.5kb-GFP mice. A non-targeting gRNA (NT1) was used as the negative control. A high efficiency of knockout was confirmed on uncloned cultures of the infected cells, as compared to cells transduced with the NT1 gRNA, by immunoblot and immunofluorescence (Fig 4 C,D,E). RT-PCR of RFX3 and of two known targets of RFX3, IFT88 and DYNC2U, were also significantly diminished by the knockout (Fig 4 F). The knockout cells were also grown in culture for $96 \mathrm{hrs}$ then stained for K8. The number of GFP+ cells in the RFX3- culture was significantly lower than in the NT1 culture, while K8+ cell numbers were increased (Fig 5 A,B,C). Moreover, when we performed time-lapse imaging and traced isolated myoepithelial cells back to when they were initially plated, fewer of the RFX3- cells became GFP+ (30\% versus $60 \%$ for the NT1 control cells), and - surprisingly - more GFP- cells transdifferentiated into the K8+ luminal lineage (Fig $5 \mathrm{D}$ ). These data suggest a reduced frequency of de-differentiation towards the cap cell lineage and/or increased transdifferentiation 198 into K8+ luminal cells. We also observed that there was a delay in the onset of GFP expression, 199 and more cells reverted to a non-GFP+ state (Fig 5 E).

200 To further investigate mechanism, we repeated the experiment using RFX3 knockout in purified 201 GFP+ cap cells. As shown in Fig 5 F,G,H the loss of RFX3 reduced the GFP+ population and 202 increased the fraction of K8+ cells. This result suggests that RFX3 normally functions to 
stabilize cap cell identity and prevent transdifferentiation into the luminal lineage. Because luminal cells are not competent to regenerate mammary glands, a prediction of this model is that deletion of RFX3 would reduce the capacity of basal cells (cap or myoepithelial cells) to form mammospheres, or to regenerate mammary glands in vivo after transplantation into the cleared fat pads of recipient mice. Indeed, we observed a significant reduction in mammosphere growth of myoepithelial cells grown in 3D culture (Fig 6 C,D). Importantly, this effect was not the result of a decreased ability of RFX3 knockout cells to proliferate, as we could detect no

210 significant difference in BrdU incorporation into these cells versus an NT1 control that expresses 211 a non-targeting gRNA (Fig 6 A,B).

212 We next tested the efficiency of mammary gland regeneration in a transplantation assay. Five,

213 ten or twenty thousand unsorted mammary epithelial cells were transplanted into the cleared fat 214 pads of isogenic recipient mice. After 8 weeks the mice were euthanized and the glands were 215 removed, fixed, and stained with Carmine Alum. Analysis of these whole mounts showed that 216 mammary gland outgrowth was substantially reduced for transplanted RFX3-negative cells 217 compared to the NT1 control (Fig 6 E,F,G). We infer that the RFX3 transcription factor functions 218 to stabilize the identities of basal cell populations, which is necessary for efficient ductal 219 regeneration.

\section{DISCUSSION}

222 It has become clear in recent years that the concept of distinct, stable cell states that change in

223 a uni-directional manner during development is incorrect, and that most cells in most animal

224 tissues are in metastable states that - often in response to stress - can revert to earlier states

225 or change lineages. For example, following injury, differentiated airway epithelial cells and 226 intestinal epithelial DIl1+ secretory progenitors can become functional stem cells (Tata et al., 227 2013; van Es et al., 2012). Injury to the heart in zebrafish results in dedifferentiation and 228 proliferation of cardiomyocytes (Jopling et al., 2010). Differentiated cells can also be forced to 229 revert to a stem cell state by the over-expression of various transcription factors, most famously

230 for mesenchymal fibroblasts, which in the context of the Yamanaka factors are reprogrammed 231 into pluripotent stem cells. Mature pancreatic acinar cells can be converted directly into beta 232 cells by expression of Ngn3, Pdx1 and Mafa transcription factors (Zhou, Brown, Kanarek, 233 Rajagopal, \& Melton, 2008); and mammary luminal cells can be driven to a multipotent stem-like cells by expression of Sox9 and Slug (Guo et al., 2012). On the other hand, mature mammary myoepithelial cells, which in vivo normally form a discrete, self-renewing population with no evidence of multipotency, can spontaneously convert to a stem-like state after isolation from the 
237 mammary gland and grown in culture, or transplanted into a recipient fat pad (Prater et al.,

238 2014; Van Keymeulen et al., 2011). This conversion suggests that the micro-environment plays

239 an important role in stabilizing the differentiated state of mature myoepithelial cells, a hypothesis

240 consistent with recent demonstrations that DNA damage to the mammary gland or the ablation

241 of luminal cells by diphtheria toxin in vivo results in transdifferentiation of myoepithelial cells to

242 the luminal lineage. However, the mechanism for this conversion remains unclear. Do

243 myoepithelial cells directly switch lineage, or do they pass through an intermediate, stem-like

244 state? And what gene regulatory network controls lineage stability versus instability?

245 To address these questions, we examined the transdifferentiation of mature mammary

246 myoepithelial cells towards the luminal lineage when they are isolated from the mammary gland

247 and grown in culture. Single cell RNAseq showed that over a period of $96 \mathrm{hrs}$, a fraction of the

248 myoepithelial cells take on a luminal identity in vitro, and cell tracking showed that the majority

249 of such cells pass through an intermediate state in which they express a marker of cap cells,

250 which are myoepithelial cell progenitors. Differential gene expression analysis and regulatory

251 network analysis using iRegulon identified several transcription factors that potentially control

252 expression of multiple genes that are upregulated in cap cells. One of these, RFX3, proved to

253 be necessary for the stability of basal (cap and myoepithelial) cell identities. Knockout of RFX3

254 strongly reduced the number of cells that expressed the cap cell GFP marker. Moreover, while

255 loss of RFX3 had no impact on proliferation of myoepithelial cells in vitro, it significantly reduced

256 mammosphere growth and reduced mammary ductal outgrowth in a transplantation assay.

257 These data suggest that RFX3 is not required for cap cell identity, or for conversion of

258 myoepithelial cells to the luminal lineage, but instead plays a role in stabilizing cell identity. We

259 speculate that state stabilization is an important process in development, and that multiple

260 transcription factors, regulated by signaling from the micro-environment, might operate in

261 different contexts and tissues to ensure phenotypic stability.

263 ACKNOWLEDGEMENTS

264 This work was supported by grant R35 CA197571 to IGM, and T32 HD07502 to EMT. We thank 265 members of the Macara laboratory for advice and support. 


\section{FIGURES AND FIGURE LEGENDS}

Figure 1.

A.
$\frac{\hat{M C}}{\mathrm{GFP}}+\frac{\hat{\mathrm{K}} 8+}{\mathrm{N}+}$

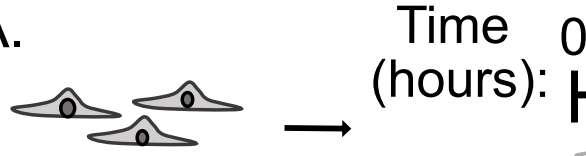 Sort for Myoepithelial Cells (MC)
72
72

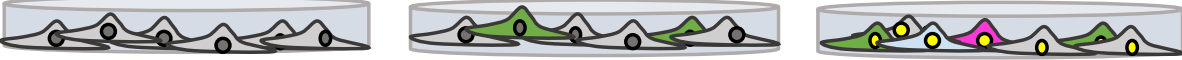 Plate onto Culture for 96 hours Fix, stain cover glass in FAD media and RI and analyze

B.

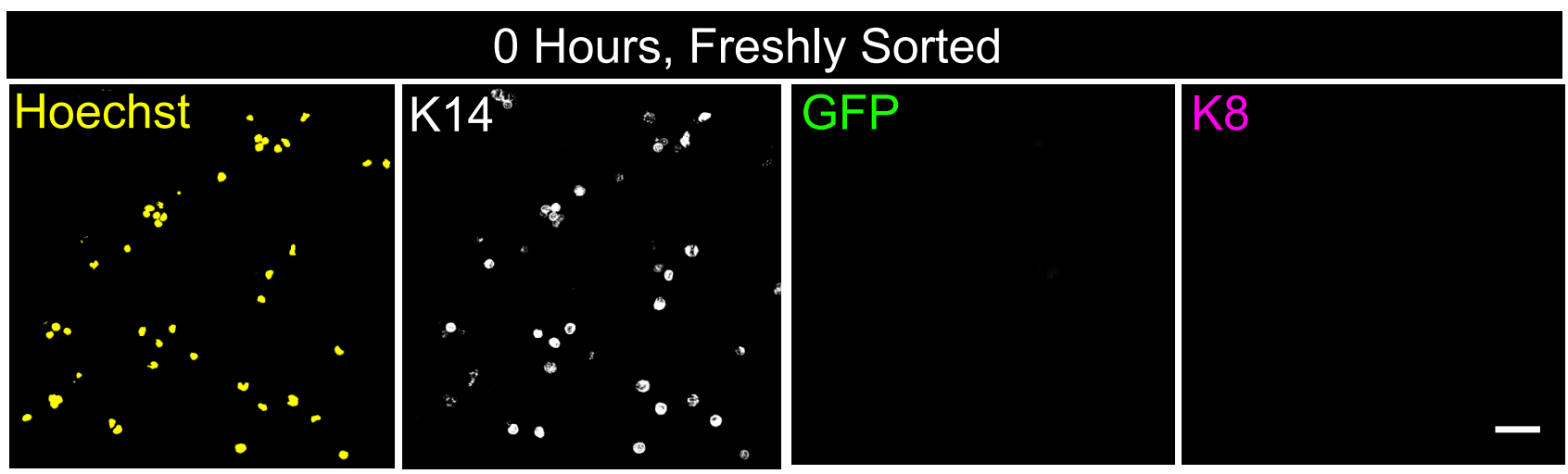

C.

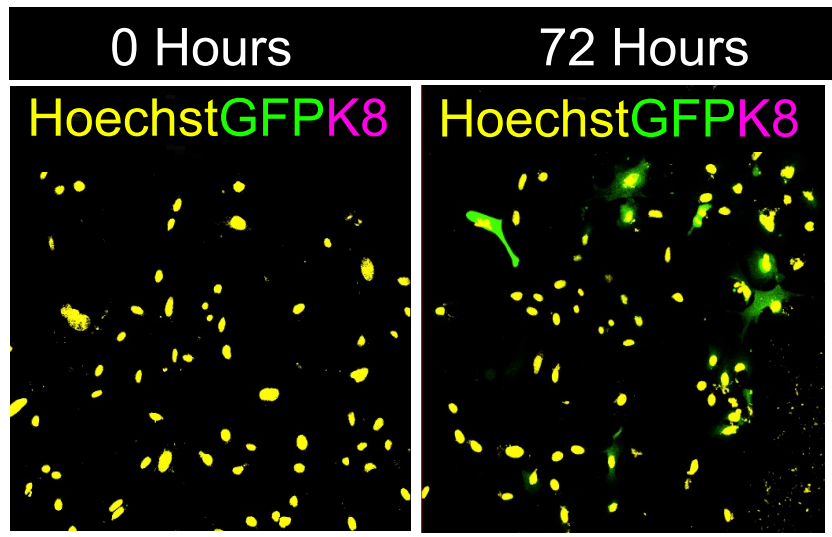

96 Hours

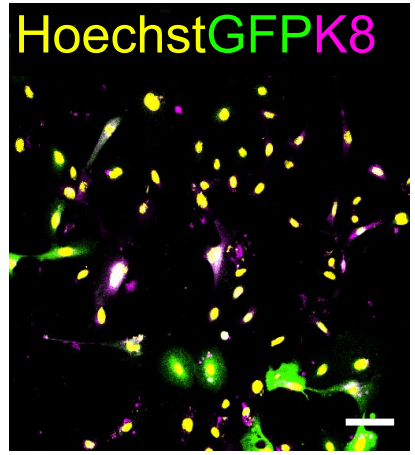

D.

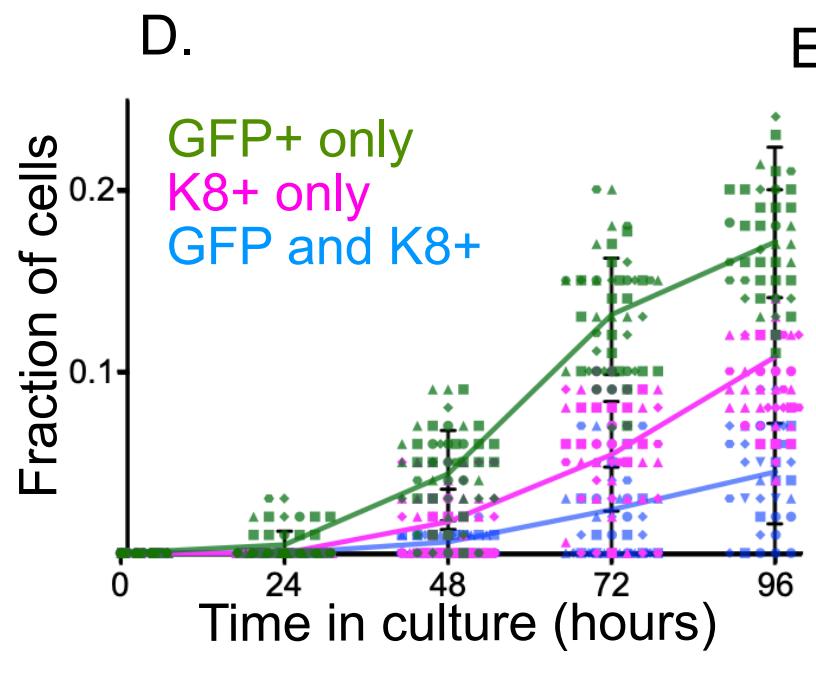

E. $\mathrm{K} 14$
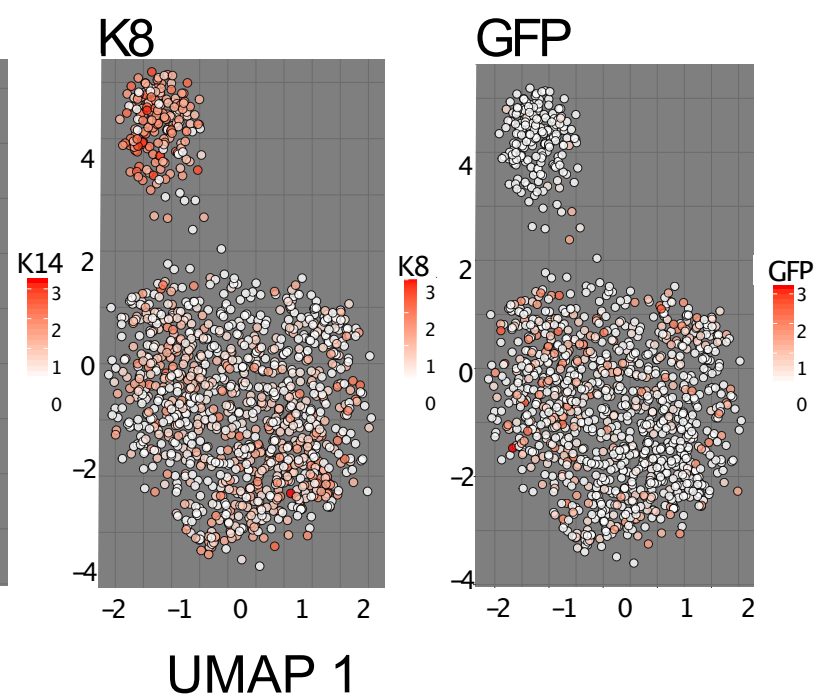

luminal marker, K8, after short-term culture

A. Diagram of experimental approach used to follow transdifferentiation of myoepithelial cells (MC) into luminal marker-expressing $\mathrm{K} 8+$ cells.

B. Representative image of freshly sorted myoepithelial cells at $0 \mathrm{hrs}$ in culture, expressing only myoepithelial cell marker K14. Scale bar $=100 \mu \mathrm{m}$

C. Representative images of myoepithelial cells cultivated in FAD medium with ROCK inhibitor Y-27632 (RI) over 96 hrs. Scale bar $=100 \mu \mathrm{m}$

D. Quantification of cap cell and luminal cell marker expression during culture of myoepithelial cells with Y-27632. A fraction of cells become GFP+, K8+ or express both markers, $n=5$ biological replicates (each a different shape), 10 fields/experiment. Error bars = Mean +/SD.

E. UMAP projections of myoepithelial cells after $96 \mathrm{hrs}$ culture. Single Cell RNA sequencing was used to observe myoepithelial cell gene expression changes over 96 hours. 
Figure 2.

A.

Image every 12 hours for 96 hours
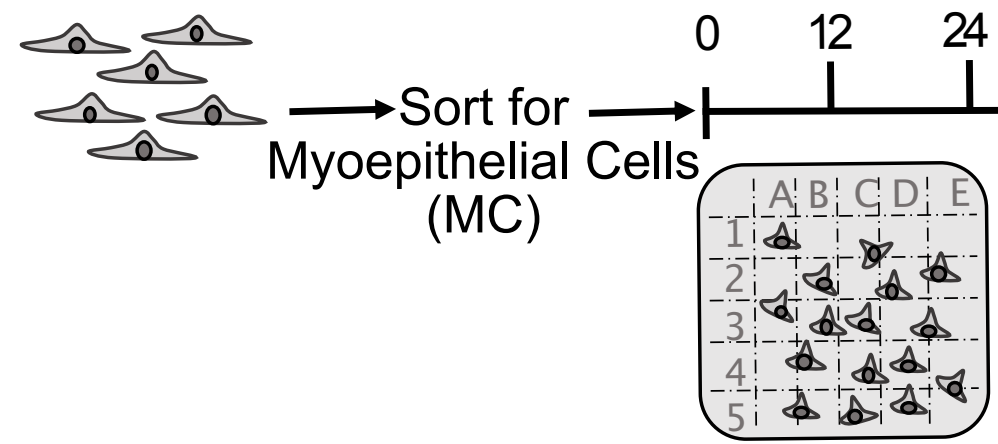

Plate onto

Culture for 96 hours
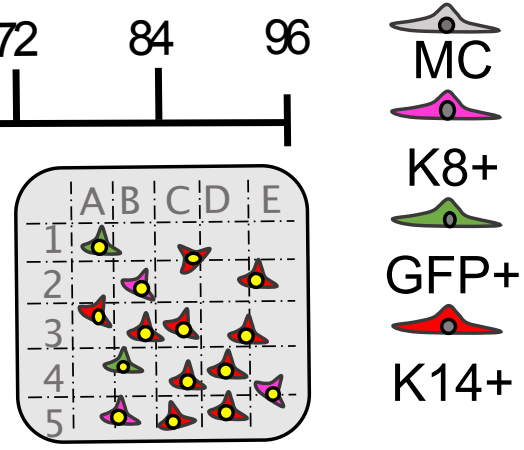

B. cover glass in FAD media and RI and analyze

\section{Hours}
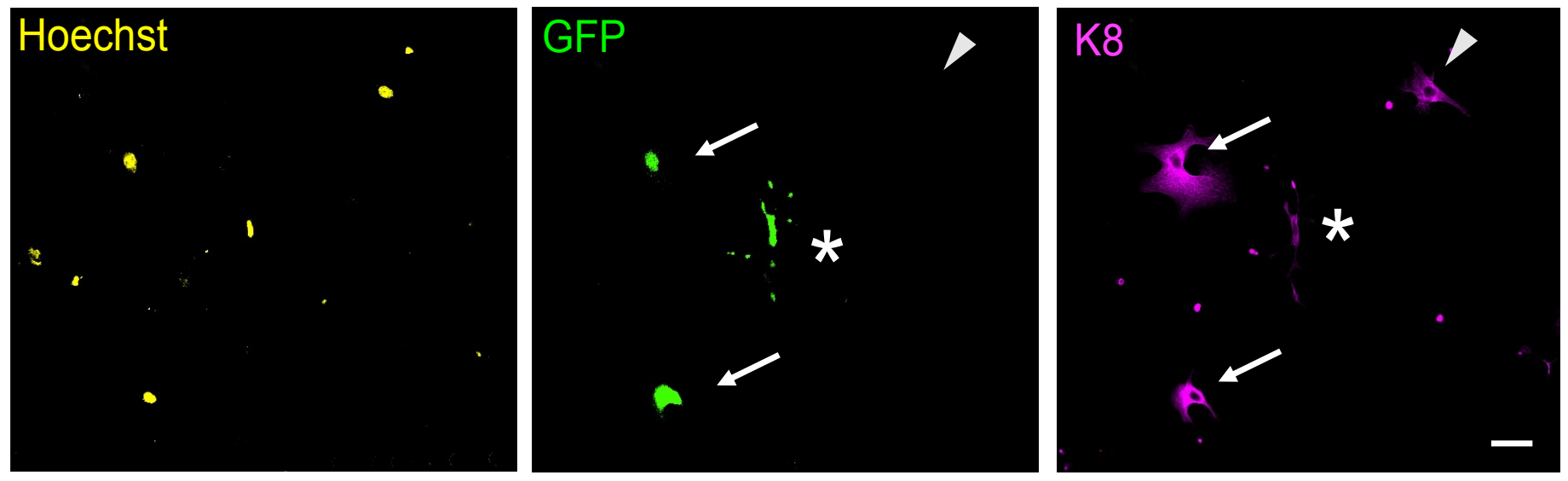

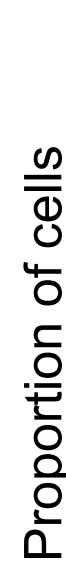

C.
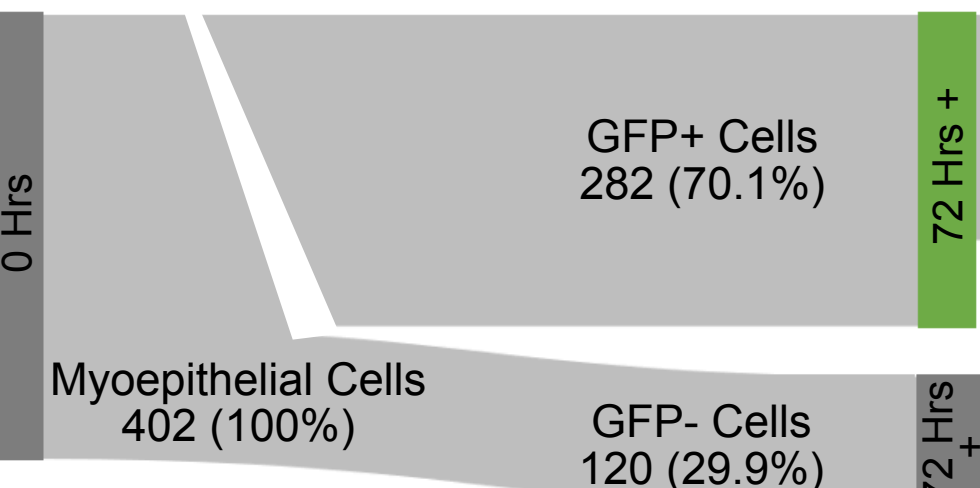

$\mathrm{K} 8+$ Cells

$240(50.7 \%)$

$\mathrm{K} 8+$ Cells

$36(8.9 \%)$

Time in culture (hours) 

prior to expressing luminal cell markers during transdifferentiation

A. Diagram of experimental approach to track transdifferentiation of single myoepithelial cell (MC) conversion in vitro toward the luminal lineage $(\mathrm{K} 8+)$.

B. Representative images of myoepithelial cell transdifferentiation at $96 \mathrm{hrs}$, and differential conversion paths. Images only showing GFP and K8 expression. Arrows indicate cells that express GFP prior to expressing luminal marker K8. Arrowheads point to cells that express K8 without ever expressing GFP, and the star indicates a cell that becomes GFP+ but did not become K8+. Scale bar $=100 \mu \mathrm{m}$.

C. Sankey Plot displaying cell gene expression decisions in vitro over time. Majority of cells 
Figure 3.
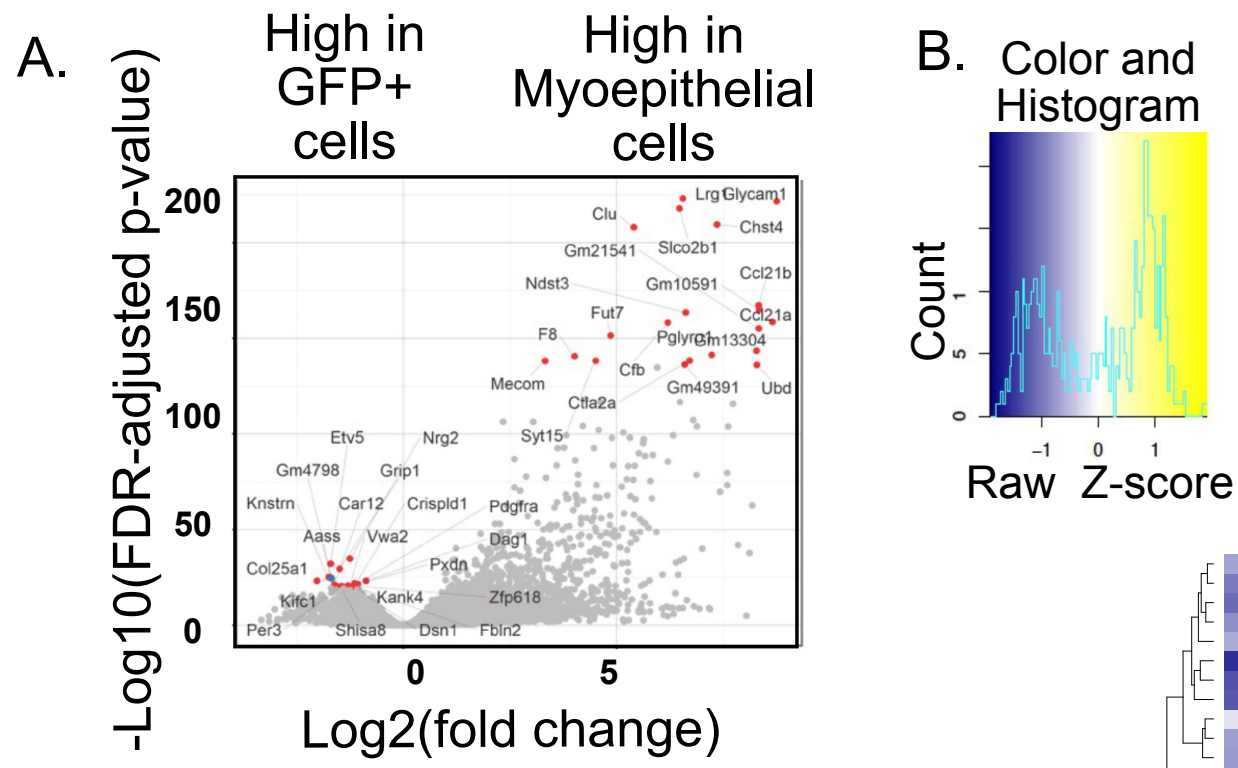

C.

Tissue Morphology

Cell. Movement

Cell. Development

Cell Growth andProlif.

Tissue Development

Embryonic Development

Organ Development

Organis. Development

Cell Func. andMaint.

Oranis. Sunvival

Protein Synthesis

Cell Morphology

Cellmed Imm. Response

Cell Death and Sunvival

Cello-CellSig. Int

Cardiovasc. Sys.

DeviFunc.

Organ Morphology

Cell Cyde

Connective Tiss.

DeviFunc.

NervousSys. Dev/Func.

Cell. Compromise

Cell Assemblyand Org.

Cell Signaling

Molecular Transp.

Skel. and Musc

Sys/Func.

Organ. Func.

Post-Trans. Mods.
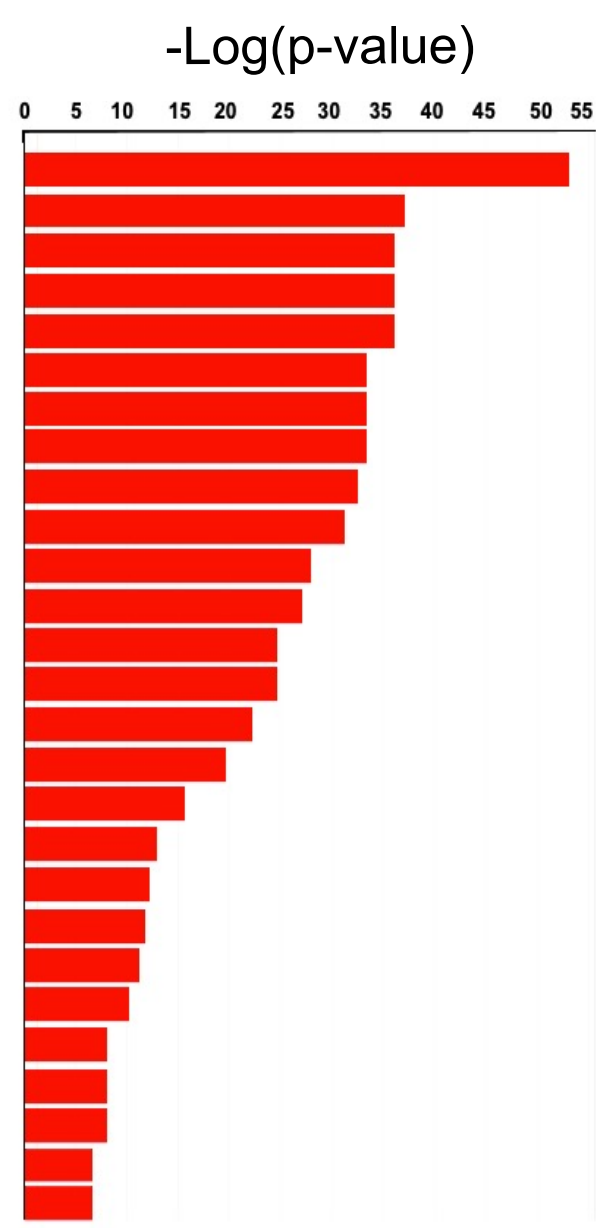

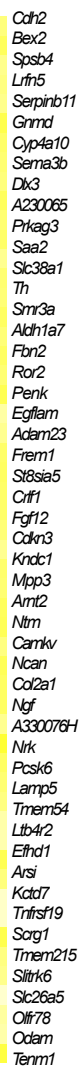

D.

Myoepithelial cells

Cap cells

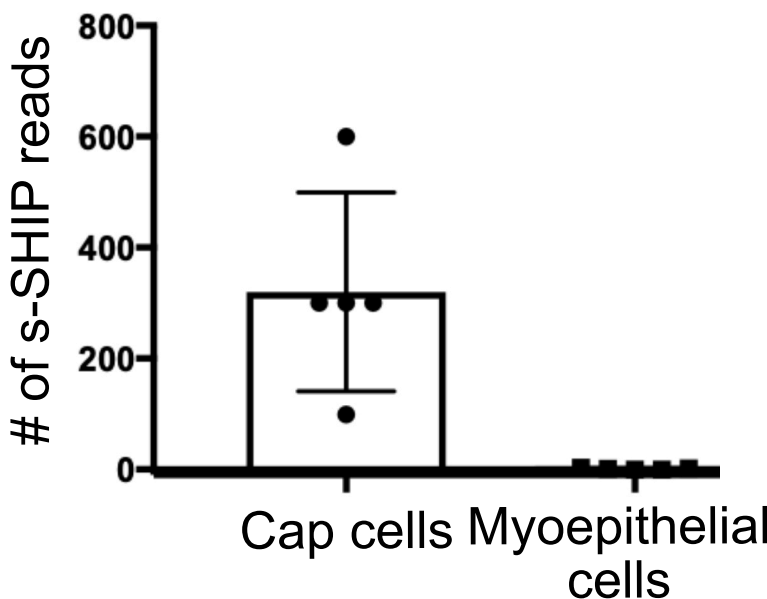


Figure 3. Differential gene expression in myoepithelial cells vs. cap cells

A. Volcano plot displaying differential gene expression with the top 20 most highly expressed genes for each sample in red. Genes filtered with a p-value $<0.005$.

B. Heatmap displaying $\sim 50$ of the top genes differentially expressed between myoepithelial cells and cap cells. Genes filtered with a p-value $<.05$ and fold change $>2$.

C. GO ontology graph of biological functions of genes most highly expressed in cap cells. Genes filtered with a p-value of $<0.05$ and fold change $>2$.

D. Dot plot displaying read count values from RNA sequencing data which show that sSHIP is differentially expressed between GFP+ cap cells and myoepithelial cells. Each dot is a biological replicate. $n=5$. Error bars $=$ Mean $+/-$ SD. 
Figure 4.

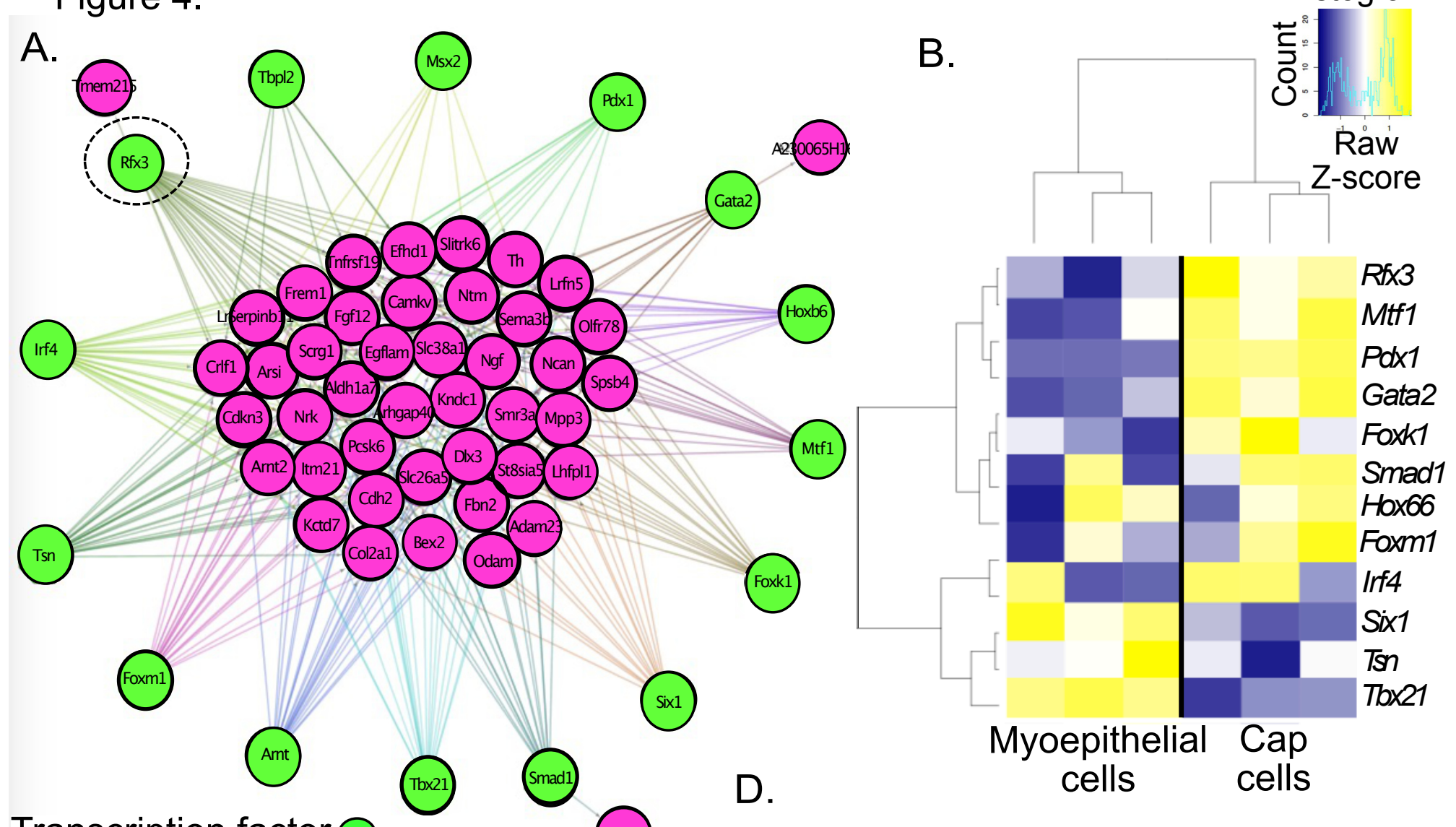

Transcription factor $\bigcirc$ Transcription factor of interest Target gene $\bigcirc$

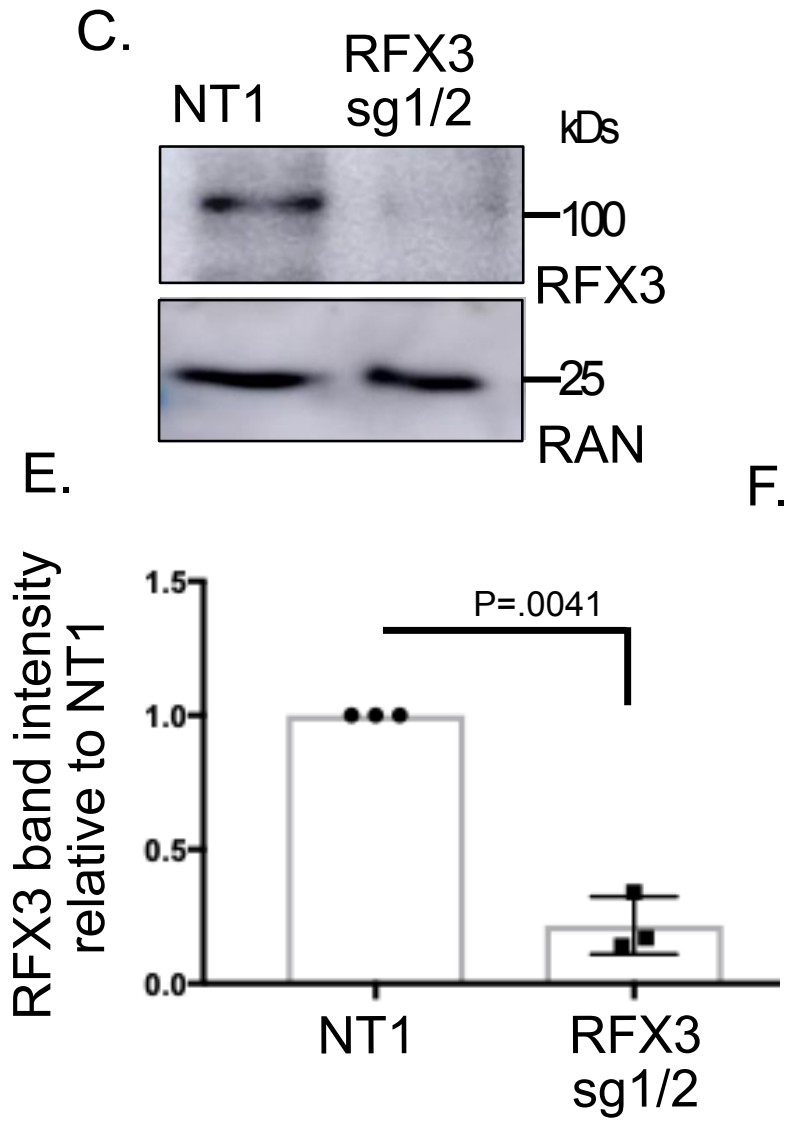

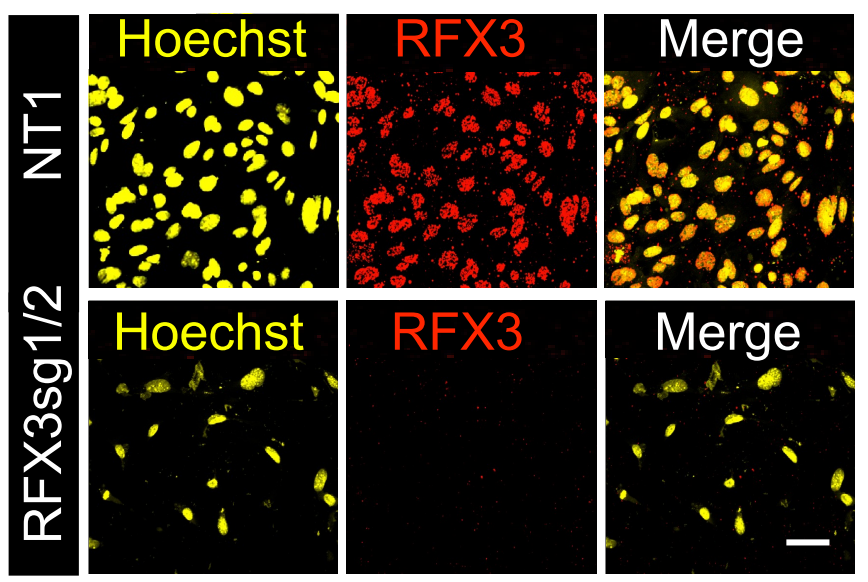
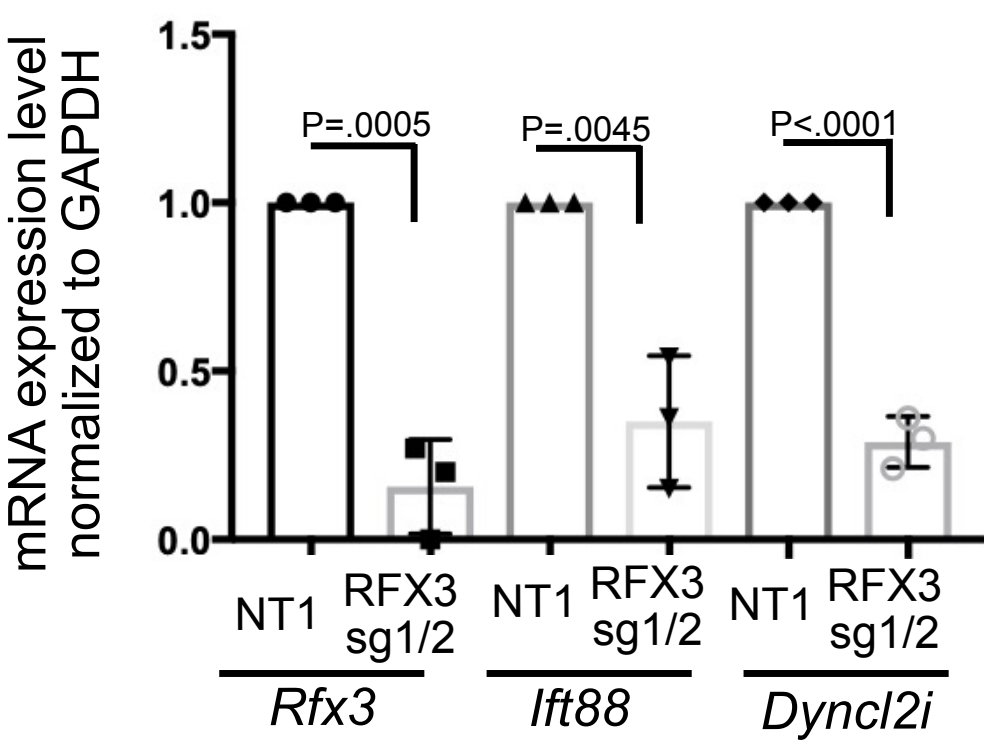
Figure 4. Gene Regulatory Network Analysis identifies Regulatory Factor X3 (RFX3) as a potential upstream regulator of cap cell gene expression

A. iRegulon Web showing the top 15 transcription factors (green) predicted to regulate the differentially expressed genes (DEGs) (pink) most highly expressed cap cells. RFX3 was a transcription factor of interest (black dashed circle).

B. Heat map of differentially expressed transcription factors predicted to regulate genes most highly expressed in cap cells.

C. Western blot confirming RFX3 KO in commaD Beta cells. anti-RAN is used as the loading control.

D. Immunocytochemistry confirming RFX3 knockout (KO) in primary cap cells. NT1 is the nontargeting 1 control lentivector and RFX3sg1/2 are the single guide lentivectors 1 and 2 used to excise the RFX3 DNA binding domain. Scale bar $=100 \mu \mathrm{m}$

F. Quantification of western blot. RFX3 band intensity relative to NT1. P-value $=.0041$, statistics calculated using unpaired t-test. Error bars = Mean +/- SD.

G. qRT-PCR confirms RFX3 loss results in a decrease in target gene (IFT88 and DYNC2LI) bars $=$ Mean $+/-$ SD. 
bioRxiv preprint doi: https://doi.org/10.1101/2021.12.13.472491; this version posted December 13, 2021. The copyright holder for this preprint (which was not certified by peer review) is the author/funder. All rights reserved. No reuse alloweo without permis $\$ p=0005$

Figure 5.

A.

Myoepithelial Cells (MC)
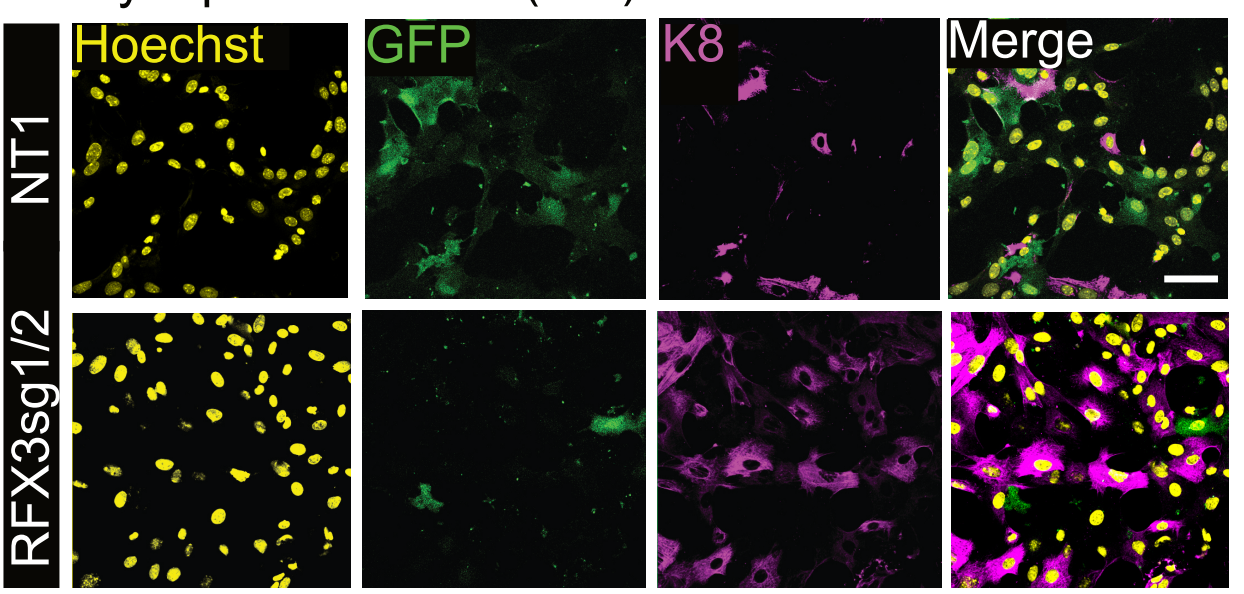

D.

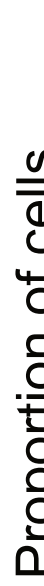

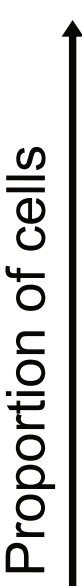
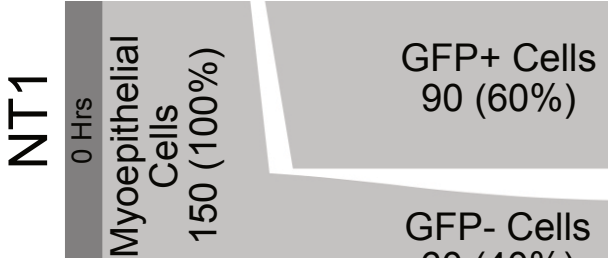
$90(60 \%)$
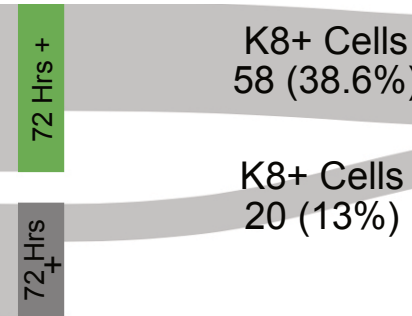

GFP-Cells

$20(13 \%)$

$\mathrm{K} 8+$ Cells

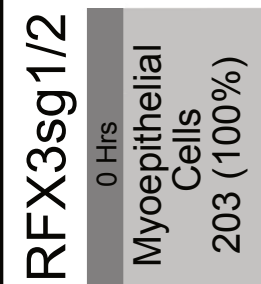

GFP+ Cells

$61(30 \%)$

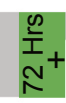

GFP- Cells

$142(69.9 \%)$

K8+ Cells

$97(47.7 \%)$

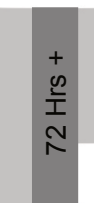

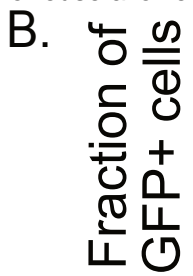

C.

E. Time of conversion (hours)

G.

F.

Time in culture (hours)

GFP+ Cells

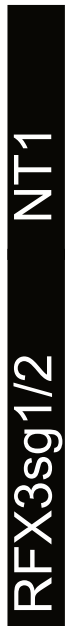

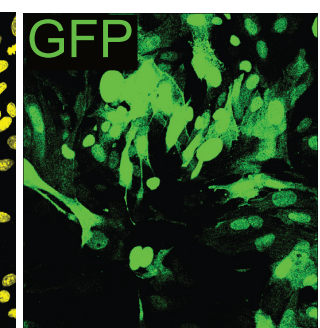
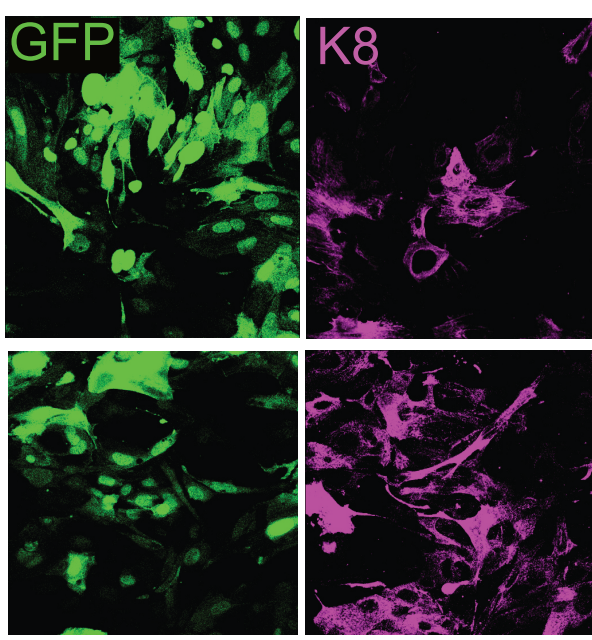
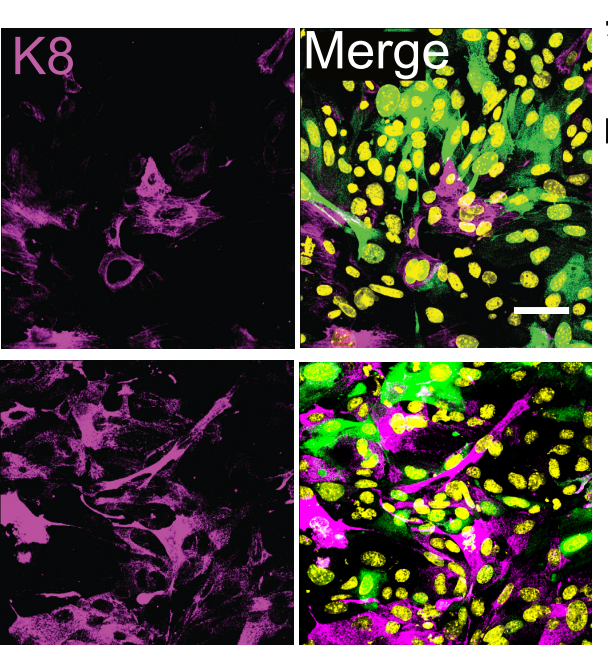
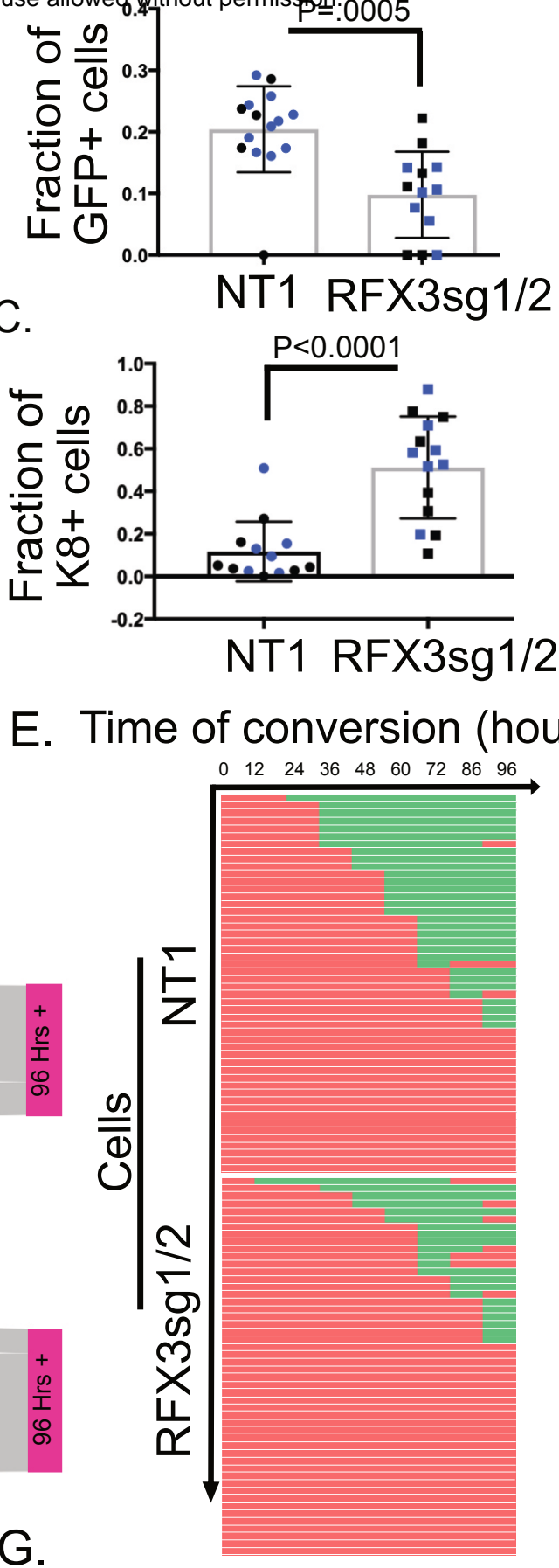

NT1 RFX3sg1/2

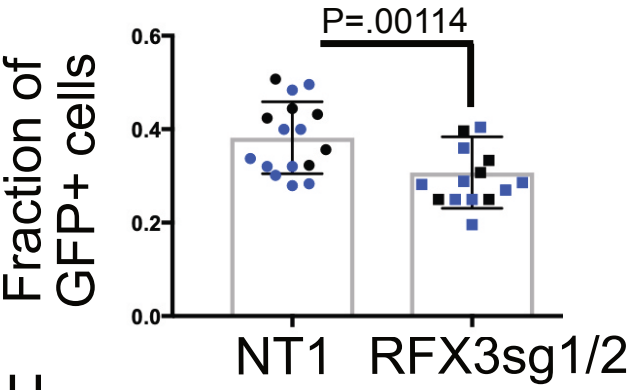

$\mathrm{H}$.

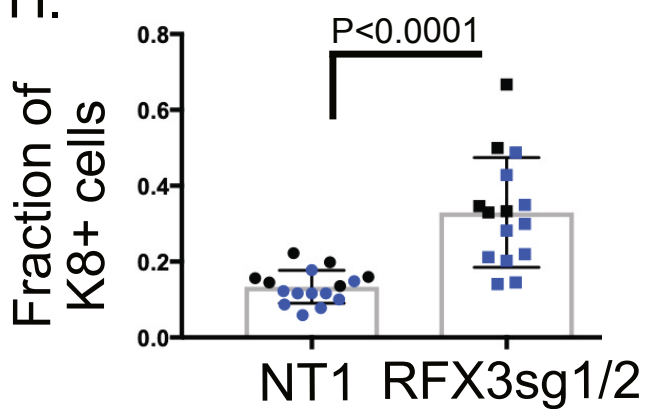


Figure 5. Loss of RFX3 promotes luminal transdifferentiation and reduces GFP+ cap cell abundance.

A. Representative images of RFX3 knockout (RFX3sg1/2) versus a negative control (nontargeting gRNA) in myoepithelial cells cultured in vitro for $96 \mathrm{hrs}$. Scale bar $=100 \mu \mathrm{m}$

B. Quantification showing a decrease in the number of myoepithelial cells that become GFP+ (NT1: $n=14,2$ experiments, RFX3sg1/2: $n=13,2$ experiments) when RFX3 is lost. P-value $=.0005$, statistics calculated using mixed model ANOVA. Error bars = Mean +/- SD.

C. Quantification graph showing an increase in the fraction of luminal cells $(\mathrm{K} 8+)$ (NT1:n= 13, 2 experiments, RFX3sg1/2:n=14, 2 experiments) that arise. Individual data points show values for technical replicates; different colors identify biological replicates. Statistics calculated using mixed model ANOVA. Error bars $=$ Mean +/- SD.

D. Sankey Plots of NT1 and RFX3 knockout (RFX3sg1/2) transdifferentiation behavior in vitro after $96 \mathrm{hrs}$. Loss of RFX3 results in a majority of myoepithelial cells converting towards a luminal cell fate, bypassing a GFP+ phase.

E. Kymograph showing times of conversion to GFP expression of myoepithelial cells. $n=50$ cells

F. Representative images of RFX3 knockout (RFX3sg1/2) or the non-targeting control in FACS-purified GFP+ cap cells cultured in vitro for $96 \mathrm{hrs}$. Scale bar $=100 \mu \mathrm{m}$

G. Quantification showing a decrease in the number of myoepithelial cells that become GFP+ (NT1: $n=16,2$ experiments, RFX3sg1/2: $n=14,2$ experiments) when RFX3 is lost. Individual data points show values for technical replicates; different colors identify biological replicates. Statistics calculated using mixed model ANOVA. Error bars = Mean +/- SD.

H. Quantification graph showing an increase in the number of luminal cells (NT1: $n=$ 16, 2 experiments, RFX3sg1/2: $n=15,2$ experiments) that arise. P-value < SD. 
Figure 6.

A.

C.
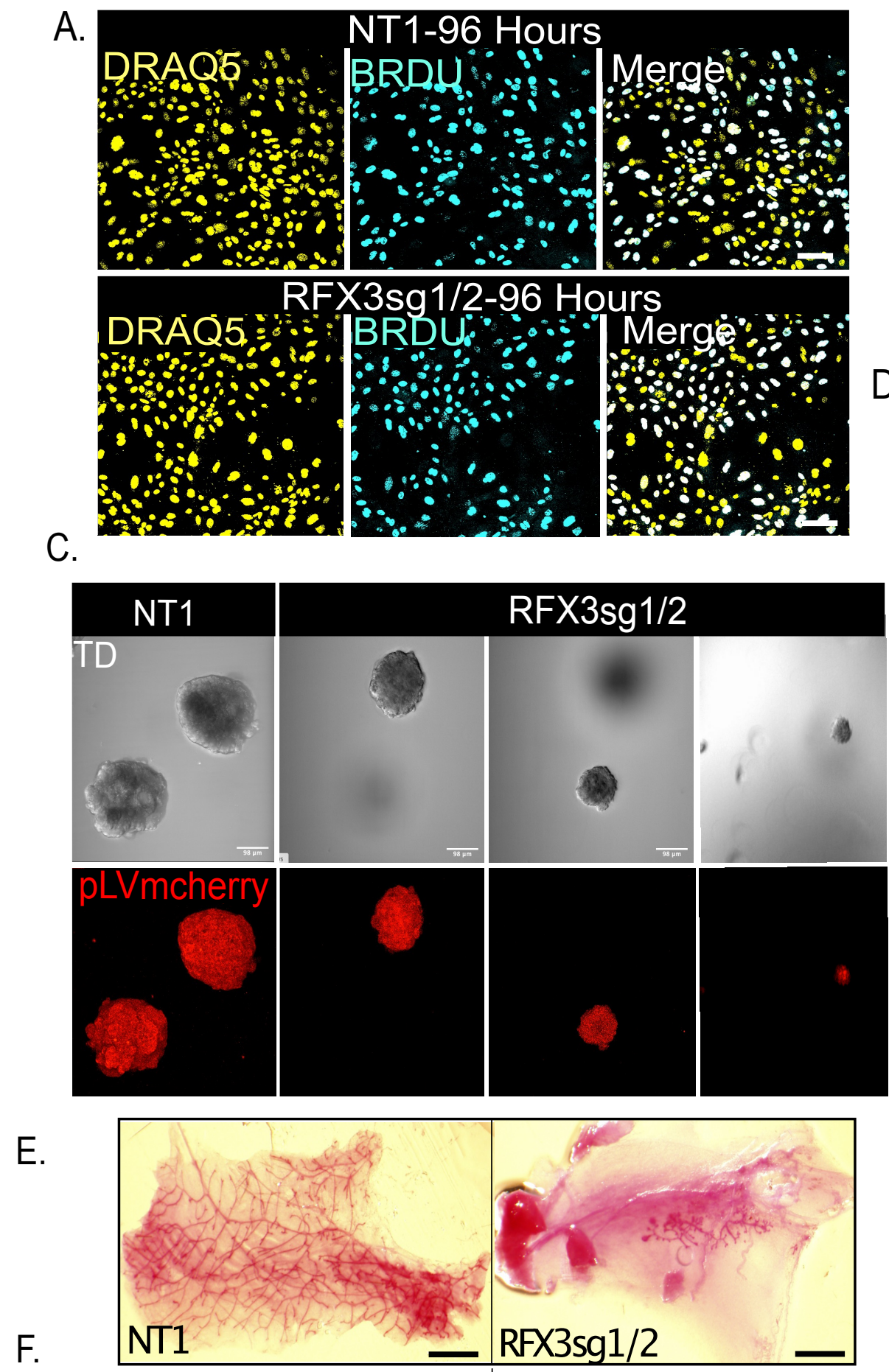

Cell \# $\quad$ NT1 $\quad$ RFX3sg1/2

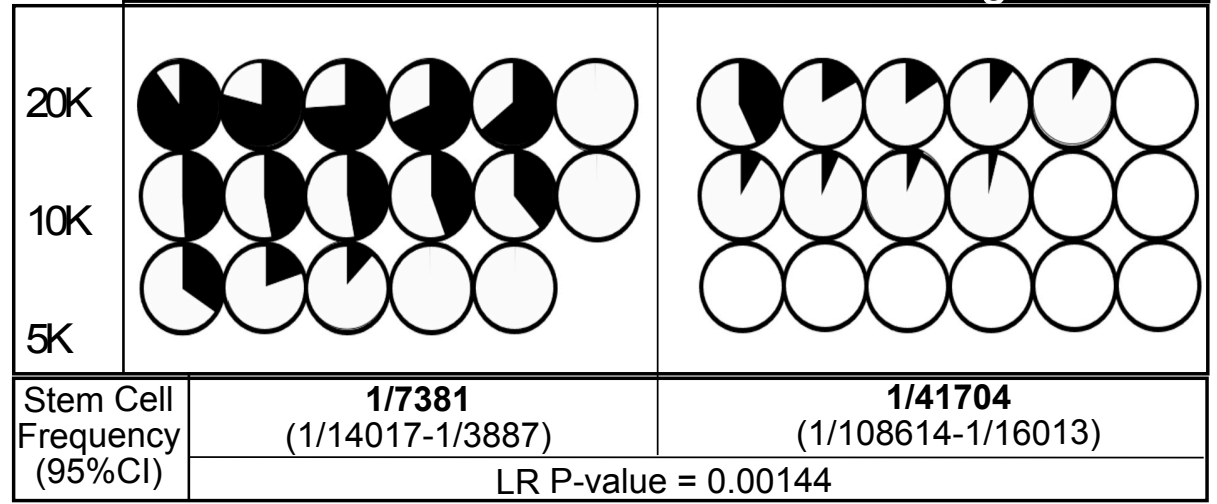

B.

n.s.

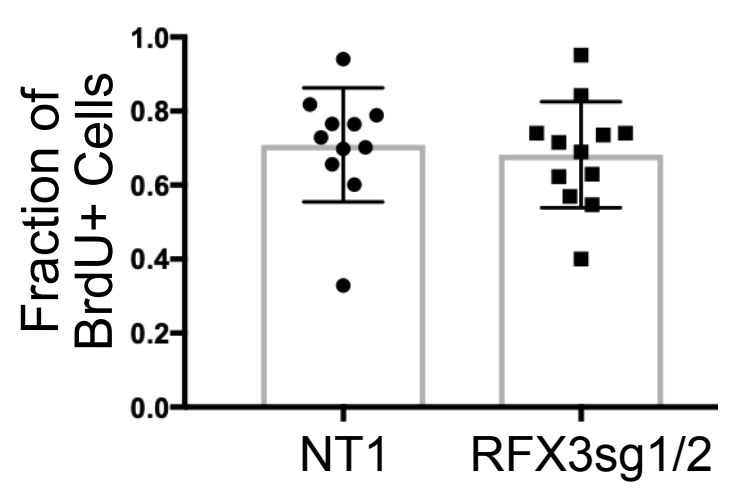

D.

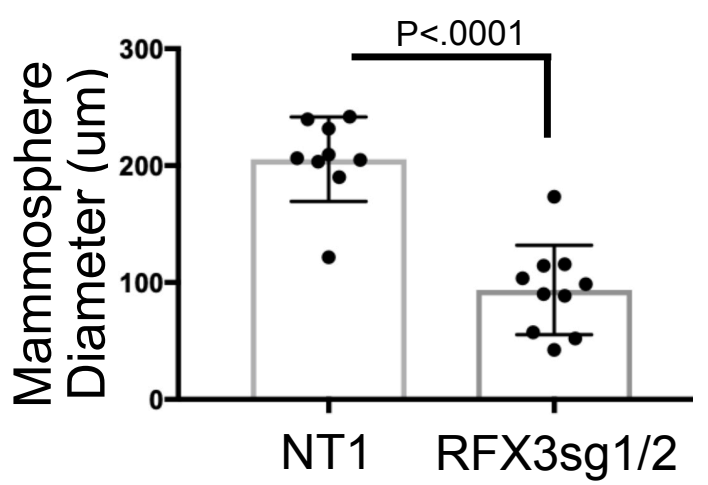

G.

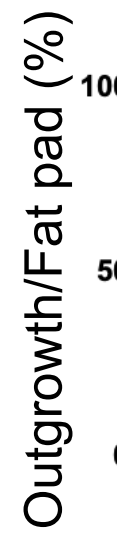

$\mathrm{H}$.

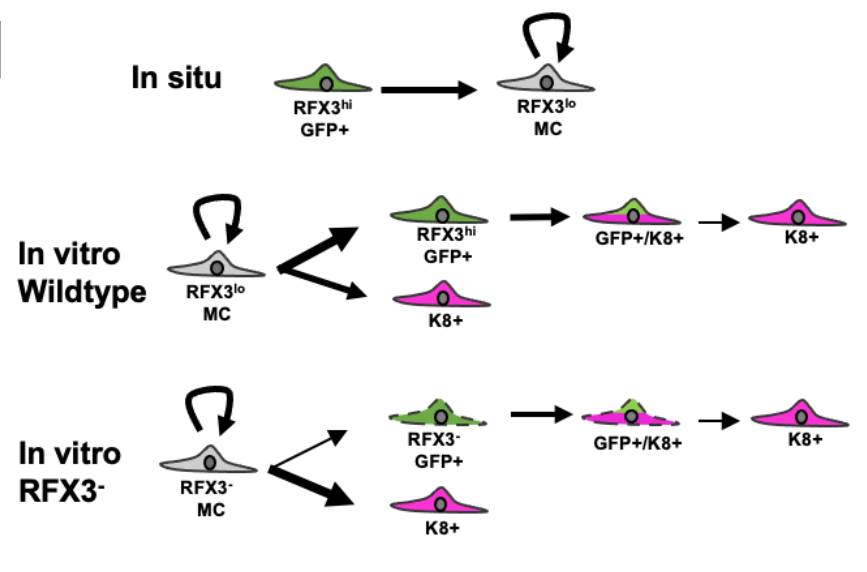


A. Representative images of myoepithelial cells transduced with either NT1 or RFX3 knockout lentivirus (RFX3sg1/2), pulse-labeled for 2 hrs with BrdU and stained for DNA (DRAQ5) and BrdU incorporation. Scale bar $=100 \mu \mathrm{m}$.

B. Quantification of fraction of BrdU+ myoepithelial cells. P-value= n.s., calculated using unpaired t-test. Error bars = Mean +/- SD.

C. Representative images of myoepithelial cells transduced with either NT1 or RFX3 knockout lentivirus (RFX3sg1/2) and grown as mammospheres. Cherry+ mammospheres confirm transduction. Scale bar $=98 \mu \mathrm{m}$

D. Quantification of mammosphere size. P-value $=<0.0001$, calculated using unpaired $t-$ test. Error bars = Mean +/- SD.

E. Representative images of outgrowths regenerated from transplantation of 20K NT1- or 20K RFX3sg1/2-transduced myoepithelial cells. Scale bar $=3 \mathrm{~mm}$.

F. Circle graphs displaying the percentage of outgrowth that resulted (outgrowth/fat pad area) from the number of cells transplanted. Stem cell frequency was calculated and generated a likelihood ratio $(L R)$ of $P$-value $=0.00144$.

G. Quantification of transplantation assay outgrowth. P-values were calculated using unpaired t-test. P-value $=$ n.s. $(5 \mathrm{~K}$ cells) 0.0018 (10K cells), 0.0082 (20K cells). Error bars $=$ Mean +/- SD.

$\mathrm{H}$. Model for the role of RFX3 in stabilizing mammary basal cell identities. In situ, cap cells, the myoepithelial progenitors (GFP+), give rise only to mature myoepithelial cells (MC) which maintain their lineage through self-renewal. In vitro, wildtype myoepithelial cells de-differentiate predominantly towards a progenitor state (GFP+) before changing lineage into K8+ luminal cells (K8). A subset of myoepithelial cells can apparently bypass the GFP+ state and transdifferentiate directly into K8+ cells. RFX3 KO myoepithelial cells have a reduced frequency of conversion into a GFP+ state (thin arrow) and increased transdifferentiation directly into a luminal cell lineage (thick arrow). 
Lead Contact

468 Further information and requests for resources and reagents should be directed to and will be

469 fulfilled by the lead contact, lan Macara (ian.g.macara@vanderbilt.edu), (615) 875-5565.

470

\section{Materials Availability}

472 This study generated Rfx3 sgRNAs (KO 1: ATCATGCAGACTTCAGAGA, KO 2:

473 GCAAGTGCCAGTGCAGCAGC) within the pLentiCRISPR-mCherry (Addgene plasmid

$474 \quad$ \#75161; http://n2t.net/addgene:75161;RRID:Addgene 75161) backbone that target the Mus

475 musculus Rfx3 (Gene ID: 19726) DNA-binding domain.

476

\section{Data and Code Availability}

478 The RNA sequencing datasets generated in this study will be deposited to the GEO repository 479 on the NCBI website. 
bioRxiv preprint doi: https://doi.org/10.1101/2021.12.13.472491; this version posted December 13,2021 . The copyright holder for this preprint (which was not certified by peer review) is the author/funder. All rights reserved. No reuse allowed without permission.

\section{KEY RESOURCES TABLE}

\begin{tabular}{|c|c|c|c|c|}
\hline \multicolumn{5}{|c|}{ Key Resources Table } \\
\hline Reagent type (species) or resource & Designation & Reagent type (species) or resource & Identifiers & Additional information \\
\hline Strain & C57BL/6J & Strain & Ca\#\#000664B6, B6J, B6/J & \\
\hline Strain & s-SHIP-GFP transgenic (Tg11.5kb-GFP) & Strain & & $\begin{array}{l}\text { Fred Hutchinson Cancer Research Center, Seattle, } \\
\text { Washington }\end{array}$ \\
\hline Antibody & Chicken polyclonal anti-Cytokeratin 14 (1:500) & Antibody & Cat\#906001; RRID: AB_2565055 & \\
\hline Antibody & Rat monoclonal anti-Cytokeratin 8 (1:500) & Antibody & Cat\#TROMA-I; RRID: AB 531826 & \\
\hline Antibody & Goat polyclonal anti-Chicken Alexa Fluor 488 (1:1000) & Antibody & Cat\#A-11039; RRID: AB 2534096 & \\
\hline Antibody & Goat polyclonal anti-Chicken Alexa Fluor 647 (1:1000) & Antibody & Cat\#A-21449; RRID: AB_2535866 & \\
\hline Antibody & Goat polyclonal anti-Rabbit Alexa Fluor 488 (1:1000) & Antibody & Cat\#A-11034; RRID: AB 2576217 & \\
\hline Antibody & Goat polyclonal anti-Rabbit Alexa Fluor 594 (1:1000) & Antibody & Cat\#A-11037; RRID: AB 2534095 & \\
\hline Antibody & Donkey polyclonal anti-Goat Alexa Fluor 594 (1:1000) & Antibody & Cat\#A32758; RRID: AB 2762828 & \\
\hline Antibody & Donkey polyclonal anti-Goat Alexa Fluor 647 (1:1000) & Antibody & Cat\#A-21447; RRID: AB 2535864 & \\
\hline Antibody & Donkey polyclonal anti-Mouse Alexa Fluor 594 (1:1000 & Antibody & Cat\#A-21203; RRID: AB 141633 & \\
\hline Antibody & Donkey polyclonal anti-Mouse Alexa Fluor 647 (1:1000 & Antibody & Cat\#A-31571; RRID: AB 162542 & \\
\hline Antibody & Donkey polyclonal anti-Rat Alexa Fluor 594 (1:1000) & Antibody & Cat\#A-21209; RRID: AB 2535795 & \\
\hline Antibody & Goat polyclonal anti-Rat Alexa Fluor 647 (1:1000) & Antibody & Cat\#A-21247; RRID: AB 141778 & \\
\hline Antibody & PE rat anti-mouse CD31 (1:200) & Antibody & CaA\#554656AB;RRID:AB_394819 & \\
\hline Antibody & PE rat anti-mouse TER-119 (1:200) & Antibody & Cat\#12-5921-82; RRID: AB_466042 & \\
\hline Antibody & PE rat anti-mouse CD45 (1:200) & Antibody & Cat\#103105; RRD: AB 312970 & \\
\hline Antibody & PB rat anti-mouse CD24 (1:400) & Antibody & Cat\#101819; RRID: AB 572010 & \\
\hline Antibody & APC rat anti-mouse CD326 (1:600) & Antibody & Cat\#50-152-15; RRID: 175791 & \\
\hline Antibody & PerCP-Cy5 rat anti-human/mouse CD49f (1:200) & Antibody & Cat\#50-166-772; RRID: AB 313618 & \\
\hline Antibody & rabbit anti-RFX3 (1:500) & Antibody & $\underline{\text { Cat\#NBP1-86301 }}$ & \\
\hline Antibody & rabbit anti-Ran13 (1:1000) & Antibody & & \\
\hline Other & Hoechst $33342(1: 1000)$ & Other & Cat\#H3570 & \\
\hline Other & anti-BrdU (1:1000) & Abcam & Cat\#Ab6326 & \\
\hline Recombinant DNA reagent (Mus musculus) & Rfx3 KO 1 & This paper & & ATCATGCAGACTTCAGAGA \\
\hline Recombinant DNA reagent (Mus musculus) & $\mathrm{Rfx} 3 \mathrm{KO} 2$ & This paper & & GCAAGTGCCAGTGCAGCAGC \\
\hline Recombinant DNA reagent (Mus musculus) & plentiCRISPRv2 puro & Addgene & Cat\#98290;RRID:Addgene_98290 & \\
\hline Recombinant DNA reagent (Mus musculus) & pLentiCRISPR-mCherry & Addgene & Ca\#75161;RRID:Addgene_75161 & \\
\hline Recombinant DNA reagent (Mus musculus) & control nontargeting (NT)sgRNA & This paper & & GCGAGGTATTCGGCTCCGCG \\
\hline Software & GraphPad Prism & GraphPad Software & RRID: SCR 002798 & https://graphpad-prism.software.informer.com/5.0/i \\
\hline Software & Fiji & Fiji & RRID: SCR 002285 & \\
\hline Software & NIS Elements & Nikon & RRID: SCR 014329 & https://www.nikoninstruments.com/Products/Software; \\
\hline Software & Ingenuity Pathway Analysis & Qiagen & RRID: SCR_002798 & http://www.ingenuity.com/products/pathways_analysis.html \\
\hline Software & CLC Workbench & Qiagen & RRID:SCR 011853 & https://digitalinsights.qiagen.com/ \\
\hline Software & SankeyMATIC & Github & https://sankeymatic.com & \\
\hline Software & $\mathrm{R}$ & The R Project & https://www.r-project.org & \\
\hline Sequence-based reagent & GAPDHF & This paper & & 5'-TCTCCACACCTATGGTGCAA-3' \\
\hline Sequence-based reagent & GAPDHR & This paper & & 5'-TGCCGTGAGTGGAGTCATA-3' \\
\hline Sequence-based reagent & GLYCAMF & This paper & & 5'-GTGCCACCATGAAATTCTTC-3' \\
\hline Sequence-based reagent & GLYCAMR & This paper & & 5'-TCTTCATGACTTCGTGATAC-3' \\
\hline Sequence-based reagent & CLUSTERINF & This paper & & 5'- GCATACCTGCATGAAGTTCTAT-3' \\
\hline Sequence-based reagent & CLUSTERINR & This paper & & 5'- GTAGAAGGGTGAGCTCTGGTTT-3 \\
\hline Sequence-based reagent & COLLAGEN11A1F & This paper & & 5'- GACTACTCAGATGGCATG-3' \\
\hline Sequence-based reagent & COLLAGEN11A1R & This paper & & 5'-ACTTCCTGGTTTCTCCTT-3' \\
\hline Sequence-based reagent & ETSVARIANTFACTOR5F & This paper & & 5'-GCAGTTTGTCCCAGATTTTCA-3 \\
\hline Sequence-based reagent & ETS VARIANTFACTOR5R & This paper & & 5' GCAGCTC CCGTTTGATCTT-3' \\
\hline Sequence-based reagent & RFX3F & This paper & & 5'-GGACAGCCGCTTTCAGAGAA-3' \\
\hline Sequence-based reagent & RFX3R & This paper & & 5'-TCTCTACAGCCCAGCAAGGA-3' \\
\hline Sequence-based reagent & IFT88F & This paper & & 5'-TGGCCAACGACCTGGAGATTAACA-3 \\
\hline Sequence-based reagent & IFT88R & This paper & & 5'-ATAGCTGCTGGCTTGGGCAAATTC-3', \\
\hline Sequence-based reagent & DYNC2LI1F & This paper & & 5'-GGTGAGCCGGAATACAGAGAA-3' \\
\hline Sequence-based reagent & DYNC2LI1R & This paper & & 5'-TGTTTGGTAGGATCTGGGACA-3'. \\
\hline Chemical compund, drug & Laminin & Milipore Sigma & Cat\#L2020; CAS: 114956-81-9 & \\
\hline Chemical compund, drug & BrdU & Millipore Sigma & Cat\#B5002; Beilstein Registry\#: 30395 & \\
\hline Chemical compund, drug & ROCK Inhibitor (Y-27632) & Sigma-Aldrich & Ca\#\#0503-1MG & \\
\hline Deposited Data & Raw and analyzed RNA sequencing data & This paper & GEO: pending & \\
\hline Cell Lines & Eph4, mouse & Dr. Jürgen Knoblich & N/A & Institute of Molecular Biotechnology, Vienna, Austria \\
\hline Cell Lines & HEK293T, human & ATTC & RRID:CVCL_0063 & \\
\hline Cell Lines & CommaD, mouse & Dr. Javier F. Medina & N/A & Baylor college of Medicine, Houston, TX \\
\hline
\end{tabular}

496

497

498 
MATERIALS AND METHODS

\section{Mice}

C57BL/6J (JAX stock \# 000664) female mice were purchased from Jackson Laboratory (Bar Harbor, ME). The s-SHIP-GFP transgenic (Tg11.5kb-GFP) mice were provided by Dr. Larry Rohrschneider from Fred Hutchinson Cancer Research Center, Seattle, Washington. Mice were housed in the Vanderbilt mouse facility with a standard 12hrs light/12hrs dark cycle and provided with normal laboratory chow and water. The mice were monitored daily by the Vanderbilt Division of Animal Care (DAC). S-SHIP-GFP primer sequences used for genotyping were as described previously (Bai and Rohrschneider, 2010). All mouse experiments were performed with approval from the Vanderbilt Institutional Animal Care and Use Committee (IACUC).

\section{Cell Lines}

517 Mouse mammary EpH4 cells were provided by Dr. Juergen Knoblich (Institute of Molecular 518 Biotechnology, Vienna, Austria). HEK293T (ATCC CRL-3216) cells were obtained from ATCC.

519 CommaD $\beta$ cells were provided by Dr. Medina (Baylor college of Medicine, Houston, TX). Cell

520 lines were cultured in Dulbecco's modified Eagle medium (Life Technologies), supplemented

521 with 10\% Fetal Bovine Serum (R\&D Systems, Minneapolis, MN) and 1X penicillin/streptomycin

522 (Life Technologies) and maintained in culture at $37^{\circ} \mathrm{C}$ with $5 \% \mathrm{CO}_{2}$. WPI, psPAX2 and pMD2.G

523 were provided by Didier Tron. LentiCRISPRv2 puro was a gift from Brett Stringer (Addgene

524 plasmid \# 98290; http://n2t.net/addgene:98290;RRID:Addgene 98290). pLentiCRISPR-

525 mCherry was a gift from Beat Bornhauser. (Addgene plasmid

$526 \quad \# 75161 ;$ http://n2t.net/addgene:75161;RRID:Addgene_75161). Guide RNAs for RFX3 DNA

527 binding-domain knock out (Key Resources Table) were cloned into plentiCRISPRv2

528 puro or pLentiCRISPR-mCherry. sgRNAs were ligated into lentivectors at

529 the BsmB/ restriction site as described in the Zhang lab protocol (Sanjana, Shalem, \&

530 Zhang, 2014; Shalem et al., 2014).

\section{Lentiviral Production}

533 Lentivirus was produced by transfecting $80 \%$ confluent $15 \mathrm{~cm}$ dishes of HEK293T cells.

534 Lentiviral plasmid, protein plasmid (pMD2.G) and packaging plasmid (psPAX2) were transfected

535 into HEK293T cells at a 1.5:1:1 ratio using calcium phosphate precipitation. Virus-conditioned 
536 medium was collected from cells $48 \mathrm{hrs}$ post transfection, stored at $-80^{\circ} \mathrm{C}$, and titered using

537 HEK293Tcells.

538

\section{Primary Cell Isolation}

540 To isolate mammary epithelial cells the third and fourth pairs of mammary glands were removed

541 from 4 to 6-week-old s-SHIP-GFP transgenic (Tg11.5kb-GFP) mice, minced with scissors and

542 digested in digestion medium (DMEM/F12, $2 \mathrm{mg} \mathrm{ml}^{-1}$ collagenase I (Roche), $5 \mathrm{mg} \mathrm{ml}^{-1}$ insulin

543 (Sigma), $100 \mathrm{U} \mathrm{ml}^{-1}$ penicillin/streptomycin) for $1 \mathrm{hr}$ at $37^{\circ} \mathrm{C}$. Epithelial organoids were collected

544 by centrifugation at $1500 \mathrm{rpm}$ for $5 \mathrm{~min}$. The cell pellet was washed for $1 \mathrm{~min}$ in $5 \mathrm{ml}$ of

545 DMEM/F12 containing DNase I and centrifuged at $1500 \mathrm{rpm}$ for $5 \mathrm{~min}$. The pellet was

546 resuspended in $5 \mathrm{ml}$ DMEM/F12 10\% fetal bovine serum followed by $15 \mathrm{sec}$ of centrifugation at

$5471500 \mathrm{rpm}$ five times. Cells were resuspended in $1 \mathrm{ml}$ of fresh trypsin/EDTA (Invitrogen) and

548 incubated at $37^{\circ} \mathrm{C}$ for $15 \mathrm{~min}$, dissociated into a single-cell suspension and passed through a

549 cell strainer (BD) to obtain a single-cell suspension of mammary gland cells. Primary cells were

550 transduced with lentivector (RFX3 KO lentivectors or NT1) by incubating for 1-4 hours at $37^{\circ} \mathrm{C}$.

\section{BrdU Incorporation Assay}

55372 hours post-transduction with lentivector (RFX3 KO or NT1), myoepithelial cells were pulse-

554 labeled for 2 hrs with BrdU (Millipore Sigma, Cat \# L2020). Cells were then stained with anti-

555 BrdU (Abcam, Cat\# Ab6326) according to the antibody protocol.

Flow cytometry, antibodies, and cell sorting

558 Single mammary epithelial cells were blocked in $5 \%$ goat serum for 5 min on ice, stained with 559 antibodies for 30 min on ice, washed, and resuspended in 1X PBS, 2mM EDTA, 2\%FBS.

560 Antibodies used were PE rat anti-mouse CD31 (1:200; BD Biosciences), PE rat anti-mouse 561 TER-119 (1:200; Invitrogen), PE rat anti-mouse CD45 (1:200; BioLegend), PB rat anti-mouse 562 CD24 (1:400; BioLegend), APC rat anti-mouse CD326 (Epcam) (1:600; Ebioscience), and 563 PerCP-Cy5 rat anti-human/mouse CD49f (1:200; BioLegend). The single live cells were gated 564 and sorted on 5-laser FACS Arialll flow cytometers.

\section{Myoepithelial Cell Conversion Assays}

567 Freshly sorted mature myoepithelial cells were plated on 16-well chambered coverglasses

568 (Grace Biol-Labs Cat. \# 112358) in FAD media supplemented with $10 \mu \mathrm{m}$ ROCK Inhibitor (Y- 
27632) (Sigma-Aldrich Cat.\# Y0503-1MG) and cultivated for 96 hrs. Media was changed every day and cells were fixed, stained and analyzed at $96 \mathrm{hrs}$.

\section{Cell Fate Mapping}

573 Myoepithelial cells were sparsely plated onto gridded coverslips (ibidi catalogue \#80826-G500)

574 in FAD media and live imaged on a Nikon A1R inverted confocal microscope (Nikon Instruments

575 Inc.) every 12 hrs for 96 hrs using 20X/0.75 NA. At 96 hrs cells were fixed and stained for K8

576 and nuclei. Cells were annotated at $24 \mathrm{hrs}$ and tracked up to hr 96. Cells that were tracked over

577 the $96 \mathrm{hrs}$ were scored for becoming GFP+ and whether the cells were expressing luminal

578 marker (K8) at hr 96. Sankey plots were generated using SankeyMATIC by Steve Bogart

579 (https://github.com/nowthis/sankeymatic/blob/main/README.md). Kymographs were generated

580 by analyzing images from $12 \mathrm{hrs}$ to $96 \mathrm{hrs}$ and marking the timepoint at which GFP expression

581 is visible and the timepoint when GFP expression turns off, if within the $96 \mathrm{hrs}$.

\section{Mammosphere Assays}

Myoepithelial cells were embedded in growth-factor-reduced Matrigel (BD) on16-well chambered coverglasses (Grace Biol-Labs Cat. \# 112358) and cultured in mammosphere medium supplemented with $10 \mu \mathrm{m}$ Y-27632 and cultivated for 10 days. Medium was changed every day and cells were fixed, stained and analyzed on day 10.

\section{Tissue Processing, Staining and Analysis}

590 For immunocytochemistry, cells were fixed in 4\% paraformaldehyde at room temperature for 15 591 min. Cells were permeabilized with $0.2 \%$ Triton X-100. For immunohistochemical analyses mammary tissue samples were cryo-embedded in O.C.T. (Fisher Scientific, Hampton, NH) and cryo-sectioned on a Leica C1950 cryostat generating $50 \mu \mathrm{m}$ sections of mammary tissue.

594 Tissue sections were fixed for $15 \mathrm{~min}$ in $4 \%$ paraformaldehyde or for $5 \mathrm{~min}$ in $-20^{\circ} \mathrm{C}$ Acetone.

595 Both cells and tissues were blocked in 1x Western Blocking Reagent (Roche). The primary 596 antibodies used in this study included: chicken a-cytokeratin 14, rabbit a-S100A4 (BioLegend, 597 San Diego, CA), rat a-cytokeratin 8 (Developmental Studies Hybridoma Bank, lowa City, lowa), 598 rabbit a-rfx3 (Novus Biologicals, Centennial, CO). Secondary antibodies used in this study 599 included: Alexa Fluor 405, 488, 594 and 647 conjugates of anti-chicken, -mouse, -rat, -rabbit 600 and -goat (ThermoFisher Scientific, Waltham, MA). Hoechst 33342 (ThermoFisher Scientific, 601 Waltham, MA) was used to stain DNA. Slides were mounted in Fluoromount G and sealed with 602 nail varnish. Laser scanning confocal Images were acquired on a Nikon A1R inverted confocal 
microscope (Nikon Instruments Inc.). 20X/0.75 numerical aperture (NA) and 40X/1.30 NA Plan Apochromat objectives were use. Type B Immersion oil (Cargille Laboratories, Cedar Grove, NJ) was used. Image post-acquisition processing was done using Nikon NIS-Elements imaging software and Fiji (ImageJ) software. The images in the manuscript are maximum intensity projections of z-stacks and merged images are composites of individual color channels.

\section{RNA Sequencing}

Total RNA (50-200ng) isolation was performed using either TRIzol (Life Technologies) or the RNeasy Mini Kit (Qiagen, Hilden, Germany). 5 samples of myoepithelial cells and 5 samples of s-SHIP GFP+ cap cells were submitted and subjected to quality analysis using a 2100 number $(\mathrm{RIN})$ value $\sim 8$. Libraries for whole transcriptome analysis were generated following Illumina's TruSeq RNA v2 sample preparation protocol. Libraries were sequenced on an Illumina HiSeq 3000 at the Vanderbilt Technologies for Advanced Genomics (VANTAGE). 150 million reads at 75 basepairs were obtained for each of the samples. Data processing, analysis and plotting were performed using R software, CLC Genomics Workbench and Ingenuity

619 Pathway Analysis (Qiagen, Hilden, Germany). Heatmaps were generated using R graphics on

620 RNAseq gene lists filtered with a p-value $<.05$ and fold change $>2$. Volcano plots were

621 generated using CLC workbench with genes filtered with a p-value of $<0.005$. The GO ontology

622 graph was generated using Ingenuity Pathway Analysis and genes were filtered with a $p$-value

623 of $<.05$ and fold change $>2$. Reads for s-Ship were quantified manually by analyzing the read

624 alignment data to the 42 nucleotide unique sequence present in this variant.

\section{Single Cell RNA Sequencing}

627 After 96 hrs cultivation, converted myoepitheial cells were dissociated using TrypLE and cell 628 viability was determined using Trypan Blue. The control sample (fresh s-SHIP GFP+ cap cells, 629 myoepithelial cells and luminal cells) and cultivated cells were encapsulated and barcoded 630 using the inDrop platform (1CellBio) with an in vitro transcription library preparation protocol 631 (Klein et al., 2015). As per Klein et al., the CEL-Seq workflow is summarized: 1) RT, 2) Exol, 3) 632 SPRI purification (SPRIP), 4) SSS, 5) SPRIP, 6) T7in vitro transcription linear Amplification, 7) 633 SPRIP, 8) RNA Fragmentation, 9) SPRIP, 10) primer ligation, 11) RT, 12) library enrichment 634 PCR. The number of cells encapsulated was calculated by the density of cells arriving at the 635 device multiplied by the duration of encapsulation. After library preparation, the samples were sequenced using Nextseq 500 (Illumina) using a 150bp paired-end sequencing kit. After 
637 sequencing, reads were filtered, sorted by their barcode of origin and aligned to the reference

638 transcriptome using the inDrops pipeline (https://github.com/indrops/indrops). Mapped reads

639 were quantified into UMl-filtered counts per gene, and barcodes that correspond to cells were

640 retrieved based on previously established methods (Klein et al., 2015).

\section{Real-time qPCR}

643 cDNA was reverse transcribed using the SuperScript III First-Strand Synthesis System

644 (Invitrogen). qPCR was performed with triplicate replicates on a BioRad CFX96 Thermocycler

645 and analyzed using the $\Delta \Delta \mathrm{Ct}$ method. Expression levels were calculated relative to GAPDH.

646 Primer sequences used were: GAPDHF 5'-TCTCCACACCTATGGTGCAA-3', GAPDHR 5'-

647 TGCCGTGAGTGGAGTCATA-3', GLYCAMF 5'-GTGCCACCATGAAATTCTTC-3', GLYCAMR

648 5'- TCTTCATGACTTCGTGATAC-3', CLUSTERINF 5'- GCATACCTGCATGAAGTTCTAT-3',

649 CLUSTERINR 5'- GTAGAAGGGTGAGCTCTGGTTT-3', COLLAGEN11A1F 5'-

650 GACTACTCAGATGGCATG-3', COLLAGEN11A1R 5'- ACTTCCTGGTTTCTCCTT-3',

651 ETSVARIANTFACTOR5F 5'- GCAGTTTGTCCCAGATTTTCA-3', ETS VARIANTFACTOR5R 5'

652 GCAGCTC CCGTTTGATCTT-3',RFX3F 5'-GGACAGCCGCTTTCAGAGAA-3', RFX3R 5'-

653 TCTCTACAGCCCAGCAAGGA-3',IFT88F 5'-TGGCCAACGACCTGGAGATTAACA-3',IFT88R

654 5'-ATAGCTGCTGGCTTGGGCAAATTC-3',

655 DYNC2LI1F 5'-GGTGAGCCGGAATACAGAGAA-3', DYNC2LI1R 5'-

656 TGTTTGGTAGGATCTGGGACA-3'.

\section{Gene Network Regulatory Analysis}

659 iRegulon (Janky et.al., 2014) was used to predict transcription factor regulation. RNA seq data 660 was filtered for genes highly expressed in GFP+ cap cells with a p-value $<.05$. Prediction data

661 was generated by surveying transcription factor binding motifs present $20 \mathrm{~kb}$ around

662 transcription start site (TSS) [TSS-10 kb,TSS+10 kb]. Gene regulatory networks were

663 generated within Cytoscape.

\section{Transplantation/ Limited Dilution Assays}

666 Myoepithelial cells were FACS sorted and transduced with either non-targeting or RFX3-

667 targeting lentivirus. 24 hrs later cells were dissociated in trypLE Select (Gibco) for 8 minutes.

668 Dilutions of $5,000,10,000$, or 20,000 cells were resuspended in 10 ul of injection medium (10

$669 \mu \mathrm{m}$ Y-27632 (Sigma-Aldrich Cat.\# Y0503-1MG), 20\% Matrigel (Corning), DMEM/F12, 40 ng/mL

670 EGF, $20 \mathrm{ng} / \mathrm{mL}$ (R\&D Systems), FGF2 (R\&D Systems) and injected into the cleared fat pads of 
671 3-week-old female C57BI6 mice (Jackson Laboratories) using a 26 gauge needle and Hamilton

672 syringe. Mice were sacrificed 8 weeks post transplantation and whole mounts analyzed.

673 Outgrowths were detected by carmine alum whole-mount. Pictures were acquired with an

674 Olympus SZX16. A positive outgrowth was described as $10 \%$ or greater percentage of

675 outgrowth/fatpad.

676

\section{Western Blots}

678 Cells were washed with 1x PBS and lysed in $1 \mathrm{ml}$ of lysis buffer containing $20 \mathrm{mM} \mathrm{HEPES}$; $\mathrm{pH}$

$6797.4,50 \mathrm{mM} \mathrm{NaCl}, 2 \mathrm{mM}$ EDTA, and 0.1\% Triton X-100, supplemented with cOmplete ${ }^{\mathrm{TM}}$ mini

680 EDTA-free protease inhibitor cocktails (Roche) and PhosStop (Roche). Cell lysates were briefly 681 centrifuged at $16,000 \times \mathrm{g}$ and the soluble fraction was boiled with SDS sample buffer for $5 \mathrm{~min}$. 682 Antibodies used for Western blotting include: rabbit anti-RFX3 (Novus Biologicals, Centennial, 683 CO) and rabbit anti-Ran13 (Macara Lab; (Richards, Lounsbury, \& Macara, 1995).

\section{Statistics}

686 All cell counts were performed using Hoechst staining and a fluorescent antigen marker in NIS-

687 Elements imaging software or Fiji (ImageJ) software. All measurements for mammosphere 688 diameter, transplant outgrowth area and western blot analysis were done using ImageJ. All 689 statistical analyses were performed using unpaired Student's t-test or two-way ANOVA test. 690 Stem cell frequencies were calculated using Extreme Limiting Dilution Analysis (ELDA) (Hu \& 691 Smyth, 2009). 


\section{REFERENCES}

706

707

708

709

710

711

712

713

714

715

716

717

718

719

720

721

722

723

724

725

726

727

728

729

730

731

732

733

734

735

736

737

738

Abdul-Manan, N., Aghazadeh, B., Liu, G. A., Majumdar, A., Ouerfelli, O., Siminovitch, K. A., \& Rosen, M. K. (1999). Structure of Cdc42 in complex with the GTPase-binding domain of the 'Wiskott-Aldrich syndrome' protein. Nature, 399(6734), 379-383.

Bai, L., \& Rohrschneider, L. R. (2010). s-SHIP promoter expression marks activated stem cells in developing mouse mammary tissue. Genes Dev, 24(17), 1882-1892. doi:10.1101/gad.1932810

Centonze, A., Lin, S., Tika, E., Sifrim, A., Fioramonti, M., Malfait, M., . . Blanpain, C. (2020). Heterotypic cell-cell communication regulates glandular stem cell multipotency. Nature, 584(7822), 608-613. doi:10.1038/s41586-020-2632-y

Chen, B., Niu, J., Kreuzer, J., Zheng, B., Jarugumilli, G. K., Haas, W., \& Wu, X. (2018). Autofatty acylation of transcription factor RFX3 regulates ciliogenesis. Proc Natl Acad Sci U S A, 115(36), E8403-E8412. doi:10.1073/pnas.1800949115

Guo, W., Keckesova, Z., Donaher, J. L., Shibue, T., Tischler, V., Reinhardt, F., ... Weinberg, R. A. (2012). Slug and Sox9 cooperatively determine the mammary stem cell state. Cell, 148(5), 1015-1028. doi:10.1016/j.cell.2012.02.008

Hinck, L., \& Silberstein, G. B. (2005). Key stages in mammary gland development: the mammary end bud as a motile organ. Breast Cancer Res, 7(6), 245-251.

Hu, Y., \& Smyth, G. K. (2009). ELDA: extreme limiting dilution analysis for comparing depleted and enriched populations in stem cell and other assays. J Immunol Methods, 347(1-2), 70-78. doi:10.1016/j.jim.2009.06.008

Humphreys, R. C., Krajewska, M., Krnacik, S., Jaeger, R., Weiher, H., Krajewski, S., . . Rosen, J. M. (1996). Apoptosis in the terminal endbud of the murine mammary gland: a mechanism of ductal morphogenesis. Development, 122(12), 4013-4022.

Janky, R., Verfaillie, A., Imrichova, H., Van de Sande, B., Standaert, L., Christiaens, V., . . . Aerts, S. (2014). iRegulon: from a gene list to a gene regulatory network using large motif and track collections. PLoS Comput Biol, 10(7), e1003731. doi:10.1371/journal.pcbi.1003731

Jopling, C., Sleep, E., Raya, M., Marti, M., Raya, A., \& Izpisua Belmonte, J. C. (2010). Zebrafish heart regeneration occurs by cardiomyocyte dedifferentiation and proliferation. Nature, 464(7288), 606-609. doi:10.1038/nature08899

Le Guelte, A. a. M. I. G. (2021). A self-limiting circuit regulates mammary cap cell plasticity through TGF-beta signaling. BioRxv. doi:https://doi.org/10.1101/2021.08.04.455067 
McDermott, K. M., Liu, B. Y., Tlsty, T. D., \& Pazour, G. J. (2010). Primary cilia regulate branching morphogenesis during mammary gland development. Curr Biol, 20(8), 731 737. doi:10.1016/j.cub.2010.02.048

Mitchell, E. H., \& Serra, R. (2014). Normal mammary development and function in mice with Ift88 deleted in MMTV- and K14-Cre expressing cells. Cilia, 3(1), 4. doi:10.1186/20462530-3-4

Prater, M. D., Petit, V., Alasdair Russell, I., Giraddi, R. R., Shehata, M., Menon, S., . . Stingl, J. (2014). Mammary stem cells have myoepithelial cell properties. Nat Cell Biol, 16(10), 942-950, 941-947. doi:10.1038/ncb3025

Richards, S. A., Lounsbury, K. M., \& Macara, I. G. (1995). The C terminus of the nuclear RAN/TC4 GTPase stabilizes the GDP-bound state and mediates interactions with RCC1, RAN-GAP, and HTF9A/RANBP1. J Biol Chem, 270(24), 14405-14411.

Rohrschneider, L. R., Custodio, J. M., Anderson, T. A., Miller, C. P., \& Gu, H. (2005). The intron $5 / 6$ promoter region of the ship1 gene regulates expression in stem/progenitor cells of the mouse embryo. Dev Biol, 283(2), 503-521. doi:10.1016/j.ydbio.2005.04.032

Sanjana, N. E., Shalem, O., \& Zhang, F. (2014). Improved vectors and genome-wide libraries for CRISPR screening. Nat Methods, 11(8), 783-784. doi:10.1038/nmeth.3047

Seldin, L., \& Macara, I. G. (2020). DNA Damage Promotes Epithelial Hyperplasia and Fate Misspecification via Fibroblast Inflammasome Activation. Dev Cell, 55(5), 558-573 e556. doi:10.1016/j.devcel.2020.09.021

Shalem, O., Sanjana, N. E., Hartenian, E., Shi, X., Scott, D. A., Mikkelson, T., . . Zhang, F. (2014). Genome-scale CRISPR-Cas9 knockout screening in human cells. Science, 343(6166), 84-87. doi:10.1126/science.1247005

Silberstein, G. B. (2001). Postnatal mammary gland morphogenesis. Microsc Res Tech, 52(2), 155-162.

Spike, B. T., Engle, D. D., Lin, J. C., Cheung, S. K., La, J., \& Wahl, G. M. (2012). A mammary stem cell population identified and characterized in late embryogenesis reveals similarities to human breast cancer. Cell Stem Cell, 10(2), 183-197. doi:10.1016/j.stem.2011.12.018

Sreekumar, A., Toneff, M. J., Toh, E., Roarty, K., Creighton, C. J., Belka, G. K., . . Rosen, J. M. (2017). WNT-Mediated Regulation of FOXO1 Constitutes a Critical Axis Maintaining Pubertal Mammary Stem Cell Homeostasis. Dev Cell, 43(4), 436-448 e436. doi:10.1016/j.devcel.2017.10.007 
772 Stingl, J., Eirew, P., Ricketson, I., Shackleton, M., Vaillant, F., Choi, D., . . Eaves, C. J. (2006). Purification and unique properties of mammary epithelial stem cells. Nature, 439(7079), 993-997.

Tata, P. R., Mou, H., Pardo-Saganta, A., Zhao, R., Prabhu, M., Law, B. M., . . Rajagopal, J. (2013). Dedifferentiation of committed epithelial cells into stem cells in vivo. Nature, 503(7475), 218-223. doi:10.1038/nature12777

van Es, J. H., Sato, T., van de Wetering, M., Lyubimova, A., Yee Nee, A. N., Gregorieff, A., .. . Clevers, H. (2012). Dll1+ secretory progenitor cells revert to stem cells upon crypt damage. Nat Cell Biol, 14(10), 1099-1104. doi:10.1038/ncb2581

Van Keymeulen, A., Rocha, A. S., Ousset, M., Beck, B., Bouvencourt, G., Rock, J., . . . Blanpain, C. (2011). Distinct stem cells contribute to mammary gland development and maintenance. Nature, 479(7372), 189-193. doi:10.1038/nature10573

Wuidart, A., Ousset, M., Rulands, S., Simons, B. D., Van Keymeulen, A., \& Blanpain, C. (2016). Quantitative lineage tracing strategies to resolve multipotency in tissue-specific stem cells. Genes Dev, 30(11), 1261-1277. doi:10.1101/gad.280057.116

Wuidart, A., Sifrim, A., Fioramonti, M., Matsumura, S., Brisebarre, A., Brown, D., . . Blanpain, C. (2018). Early lineage segregation of multipotent embryonic mammary gland progenitors. Nat Cell Biol, 20(6), 666-676. doi:10.1038/s41556-018-0095-2 of adult pancreatic exocrine cells to beta-cells. Nature, 455(7213), 627-632. 